DRAFT VERSION NOVEMBER 8, 2018

Preprint typeset using $\mathrm{LT}_{\mathrm{E}} \mathrm{X}$ style emulateapj v. 5/2/11

\title{
THE DISTRIBUTION OF ALPHA ELEMENTS IN ANDROMEDA DWARF GALAXIES
}

\author{
Luis C. VArgas ${ }^{1}$, MARla C. GeHA $^{1}$, AND ERIK J. TOLLERUd ${ }^{1, \dagger}$ \\ ${ }^{1}$ Department of Astronomy, Yale University, 260 Whitney Ave., New Haven, CT 06511, USA; luis.vargas@ yale.edu \\ Draft version November 8, 2018
}

\begin{abstract}
We present alpha to iron abundance ratios for 226 individual red giant branch stars in nine dwarf galaxies of the Andromeda (M31) satellite system. The abundances are measured from the combined signal of $\mathrm{Mg}, \mathrm{Si}$, $\mathrm{Ca}$, and Ti lines in Keck/DEIMOS medium-resolution spectra. This constitutes the first large sample of alpha abundance ratios measured in the M 31 satellite system. The dwarf galaxies in our sample exhibit a variety of alpha abundance ratios, with the average values in each galaxy ranging from approximately solar $([\alpha / \mathrm{Fe}] \sim$ $+0.0)$ to alpha-enhanced $([\alpha / \mathrm{Fe}] \sim+0.5)$. These variations do not show a correlation with internal kinematics, environment, or stellar density. We confirm radial gradients in the iron abundance of two galaxies out of the five with sufficient data (NGC 185 and And II). There is only tentative evidence for an alpha abundance radial gradient in NGC 185 . We homogeneously compare our results to the Milky Way classical dwarf spheroidals, finding evidence for wider variation in average alpha abundance. In the absence of chemical abundances for the M 31 stellar halo, we compare to the Milky Way stellar halo. A stellar halo comprised of disrupted M31 satellites is too metal-rich and inconsistent with the Milky Way halo alpha abundance distribution even if considering only satellites with predominantly old stellar populations. The M31 satellite population provides a second system in which to study chemical abundances of dwarf galaxies and reveals a wider variety of abundance patterns than the Milky Way.

Subject headings: galaxies: abundances — galaxies: dwarf — galaxies: evolution — galaxies: M31 — Local Group
\end{abstract}

\section{INTRODUCTION}

The alpha to iron abundance ratio correlates with the star formation timescale of a stellar population, its star formation history, and initial mass function (Tinsley 1979). The vast majority of stellar alpha element abundances measured outside of the Milky Way (MW) have been limited to the MW's nearby satellite galaxies. Spectroscopic studies have shown that the ratio of alpha to iron abundances in MW dwarf galaxies decrease gradually from super solar (alpha-enhanced) values at low metallicities to solar or sub-solar values at higher metallicities (e.g., Shetrone et al. 2001; Geisler et al. 2005; Letarte et al. 2010; Cohen \& Huang 2010; Kirby et al. 2011). Based only on the MW dwarf galaxy population, the characteristic (or average) stellar abundance properties seem to depend on galaxy mass and/or luminosity. The majority of the stars in the more massive dwarf galaxies have solar/sub-solar alpha abundances (e.g., Pompéia et al. 2008; Letarte et al. 2010; McWilliam et al. 2013). In contrast, less luminous dwarf galaxies tend to have a significant fraction of stars with higher alpha abundances (e.g., the Sculptor dwarf galaxy, see Starkenburg et al. 2013). The dependence on galaxy properties is also exemplified by the correlation between stellar mass and average stellar metallicity (e.g. Dekel \& Woo 2003; Grebel et al. 2003; Kirby et al. 2013).

The alpha abundance patterns of MW dwarf satellites have been interpreted as due to long star formation timescales (e.g., Carigi et al. 2002; Lanfranchi \& Matteucci 2007). Type II supernovae (SNe) begin exploding almost immediately after the onset of star formation and their ejecta have large yields of alpha elements (Woosley \& Weaver 1995; Chieffi \& Limongi 2004; Nomoto et al. 2006). Their high alpha abundance

\footnotetext{
$\dagger$ Hubble Fellow

${ }^{1}$ We will often refer to the alpha to iron abundance ratio, $[\alpha / \mathrm{Fe}]$, simply as the alpha abundance, and to the iron abundance, $[\mathrm{Fe} / \mathrm{H}]$, as the metallicity.
}

patterns are imprinted in the most metal poor stars observed in the MW dwarf galaxies (e.g., Frebel et al. 2010; Cohen \& Huang 2010). In contrast, the onset of Type Ia SNe is delayed relative to Type II SNe, and these $\mathrm{SNe}$ continue to occur long after the onset of star formation (e.g., Maoz et al. 2012; Matteucci et al. 2009). Type Ia SNe release large quantities of iron (e.g,,Iwamoto et al. 1999), thus lowering $[\alpha / \mathrm{Fe}]$ in the ISM. This occurs only after significant chemical evolution, thus producing the characteristic trend towards lower alpha abundances at higher metallicities. Dwarf galaxies with large fractions of low alpha stars thus formed the bulk of their stellar population in timescales comparable to or longer than the characteristic timescale for Type Ia SNe (Matteucci et al. 2009). Indeed, the more massive MW dwarf galaxies (e.g., Fornax and Leo I) are consistent with having a large fraction of intermediate age populations (e.g., Gallart et al.|1999; Hernandez et al. 2000; de Boer et al. 2012a; del Pino et al. 2013). In contrast, stellar systems dominated by enhanced alpha abundances reflect a large contribution from Type II SNe. Thus, they formed the bulk of their stellar population in bursts with sustained high rates of star formation thus softening the decrease in alpha abundances due to the Type Ia contribution (Gilmore \& Wyse 1991). Alternatively, star formation tapered off (or ceased completely) in a time shorter than the characteristic Type Ia timescale, due to either internal or environmental processes.

In contrast to the MW dwarf galaxies, our knowledge of the chemistry of the dwarf satellites of Andromeda (M31) has been limited to their overall iron abundance. The M 31 dwarf galaxies appear to follow a luminosity-metallicity relationship similar to the MW dwarf satellites (Kalirai et al. 2010; Kirby et al. 2013; Collins et al. 2013; Ho et al. 2014). This relation appears to be universal amongst Local Group dwarf galaxies (Kirby et al. 2013). Environment often plays a role in galaxy evolution, and it is known that the M 31 sys- 
Table 1

Observed Properties of M 31 Satellite Sample

\begin{tabular}{|c|c|c|c|c|c|c|c|c|c|c|}
\hline Galaxy & $\mathrm{M}_{V}^{a}$ & $\sigma_{\text {los }}^{b}[\mathrm{~km} / \mathrm{s}]$ & $\mathrm{V}_{r m s}^{c}[\mathrm{~km} / \mathrm{s}]$ & $\mu_{V}^{d}\left[\mathrm{mag} / \operatorname{arcsec}^{2}\right]$ & $\mathrm{r}_{1 / 2}^{d} \quad[\operatorname{arcmin}]$ & $\mathrm{PA}^{d}\left[{ }^{0}\right]$ & $\epsilon^{d}$ & $(m-M)_{0}^{e}$ & $N([\alpha / \mathrm{Fe}])^{\mathrm{f}}$ & $N([\mathrm{Fe} / \mathrm{H}])^{\mathrm{g}}$ \\
\hline M 32 & $-16.4 \pm 0.2$ & $29.9 \pm 3.0$ & $31.5 \pm 2.9$ & $11.1 \pm 0.0$ & $0.5 \pm 0.1$ & 159.00 & 0.25 & $24.5 \pm 0.2$ & 3 & 32 \\
\hline NGC 185 & $-14.8 \pm 0.1$ & $24.0 \pm 1.0$ & $28.3 \pm 2.8$ & $20.8 \pm 0.0$ & $1.5 \pm 0.0$ & 35.00 & 0.15 & $24.0 \pm 0.1$ & 71 & 299 \\
\hline NGC 147 & $-14.6 \pm 0.1$ & $16.0 \pm 1.0$ & $23.3 \pm 1.6$ & $21.2 \pm 0.0$ & $2.0 \pm 0.0$ & 25.00 & 0.41 & $24.3 \pm 0.1$ & 7 & 184 \\
\hline And VII & $-13.3 \pm 0.3$ & $13.0 \pm 1.0$ & $13.0 \pm 1.0$ & $23.2 \pm 0.2$ & $3.5 \pm 0.1$ & 94.00 & 0.13 & $24.4 \pm 0.1$ & 29 & 90 \\
\hline And II & $-12.4 \pm 0.2$ & $7.8 \pm 1.1$ & $13.4 \pm 2.1$ & $24.5 \pm 0.2$ & $6.2 \pm 0.2$ & 46.00 & 0.10 & $24.0 \pm 0.1$ & 56 & 248 \\
\hline And I & $-11.8 \pm 1.0$ & $10.2 \pm 1.9$ & $10.2 \pm 1.9$ & $24.7 \pm 0.2$ & $3.1 \pm 0.3$ & 22.00 & 0.22 & $24.3 \pm 0.1$ & 7 & 31 \\
\hline And III & $-10.2 \pm 0.3$ & $9.3 \pm 1.4$ & $9.3 \pm 1.4$ & $24.8 \pm 0.2$ & $2.2 \pm 0.2$ & 136.00 & 0.52 & $24.3 \pm 0.1$ & 8 & 35 \\
\hline And V & $-9.6 \pm 0.3$ & $10.5 \pm 1.1$ & $10.5 \pm 1.1$ & $25.3 \pm 0.2$ & $1.4 \pm 0.2$ & 32.00 & 0.18 & $24.4 \pm 0.1$ & 40 & 80 \\
\hline And X & $-7.4 \pm 0.1$ & $6.4 \pm 1.4$ & $6.4 \pm 1.4$ & $26.3 \pm 1.1$ & $1.3 \pm 0.1$ & 46.00 & 0.44 & $24.1 \pm 0.1$ & 5 & 16 \\
\hline
\end{tabular}

${ }^{a}$ Absolute magnitude $\left(\mathrm{M}_{V}\right)$ sources: And X (Brasseur et al. 2011), And I,III,V,VII (Tollerud et al.|2012), And II, NGC 147, NGC 185, M 32 (McConnachie 2012, and references therein).

${ }^{b}$ Velocity dispersion sources: And I,III,V,VII,X (Tollerud et al. 2013), And II (Ho et al.|2012), NGC 147,185 (Geha et al. 2012), M 32 (Howlev et al. 2013).

${ }^{\mathrm{c}} \mathrm{V}_{\text {rms }}$ calculated as quadrature sum of dispersion and rotation velocity; non-zero rotation velocities taken from same sources as velocity dispersions.

${ }^{\mathrm{d} S u r f a c e}$ brightness $\left(\mu_{V}\right)$, half-light radius $\left(\mathrm{r}_{1 / 2}\right)$, position angle (PA), and ellipticity $(\epsilon)$ from compilation by McConnachie (2012).

${ }^{\mathrm{e}}$ Distance moduli sources: (Conn et al. 2012), except And VII (Tollerud et al. 2012), And X (Brasseur et al. 2011), M 32 (McConnachie 2012).

${ }^{\mathrm{f}}$ Number of measurements of $[\alpha / \mathrm{Fe}]$ excluding $[\mathrm{Fe} / \mathrm{H}]>-0.5$ stars (see discussion in $\S 3.2$ All measurements are from $\mathrm{S} / \mathrm{N} \geq 15 \AA^{-1}$.

${ }^{\mathrm{g}}$ Number of measurements of $[\mathrm{Fe} / \mathrm{H}]$ used for computing metallicity radial gradients. All measurements are from $\mathrm{S} / \mathrm{N} \geq 8 \AA^{-1}$.

tem presents interesting contrasts to the MW. The M 31 dwarf galaxy population includes more massive dwarf spheroidals, and a larger overall number of massive satellites (Martin et al. 2013), with a broad range of kinematic properties (Geha et al. 2010; Tollerud et al. 2012, 2013; Collins et al. 2013). M31 has very likely experienced a more active accretion history than the MW as evidenced by the properties of its stellar halo (e.g., Brown et al. 2008; McConnachie et al. 2009; Gilbert et al. 2012). Measurements of alpha abundances in M31 dwarf galaxies can thus help elucidate the role of environment via a comparison of the alpha abundance pattern of its dwarf galaxies, relative to those of the MW satellites.

Alpha abundances in dwarf galaxies are also relevant for understanding hierarchical formation. In $\Lambda \mathrm{CDM}$, stellar halos are generally believed to have formed from accreted dwarf galaxies (Bullock \& Johnston 2005; Bell et al. 2008; Cooper et al. 2010; Font et al. 2011). The average alpha abundances of MW dwarf galaxies disagree with the abundances characteristic of the MW stellar halo (Venn et al. 2004). Robertson et al. (2005) pointed out that the population of present-day satellites need not resemble the properties of already accreted satellites, which were likely more massive and had shorter star formation timescales. However, the contribution of present-day dwarf galaxies to the stellar mass of a MW-mass halo may vary stochastically and be more important in other systems (Cooper et al. 2010). Thus, the chemical inventory in present day dwarf galaxies may be useful for informing simulations of stellar halo formation.

In this paper, we present a comprehensive study of the alpha abundance of nine dwarf galaxies in the M31 system, using Keck/DEIMOS medium-resolution spectroscopy of individual red giant branch stars (RGBs). These data constitute the first published alpha abundance for dwarf galaxies in the M 31 system. We describe our data sample in $\S 2$. We explain our chemical abundance technique in $\$ 3$, focusing in particular in those aspects of the analysis relevant to low signal to noise data. We present our results in $\S 4$ and discuss our results in the context of dwarf galaxy evolution and $\Lambda \mathrm{CDM}$ in $\$ 5$.

\section{OBSERVATIONS AND DATA REDUCTION}

We measure metallicities and alpha abundances for a large sample of stars in nine dwarf satellites of M31. The sample consists of RGB stellar spectra taken with Keck/DEIMOS (Faber et al. 2003) during multiple runs. We include data for
NGC 147, NGC 185 (Geha et al. 2010), And II (Ho et al. 2012), And VII (Ho et al. 2014), and new observations for And $\mathrm{V}$ and And $\mathrm{X}$ (described in $\$ 2.3$ ). The remainder of the spectra were drawn from the Spectroscopic and Photometric Landscape of Andromeda's Stellar Halo (SPLASH) project, in particular, the dataset presented by Tollerud et al. (2012). Table 1 lists the dwarf galaxies analyzed, the kinematic, structural parameters, and distances assumed throughout this work, and the number of alpha and iron abundance measurements per galaxy.

\subsection{Data Reduction}

The full dataset consists of medium-resolution $(\mathrm{R} \sim 6,000)$ Keck/DEIMOS spectra, taken with the $1200 \mathrm{l} / \mathrm{mm}$ grating and OG550 order blocking filter, a central wavelength of $\sim 7,500 \AA$, and either the $0 . " 7$ or $1 . " 0$ slits. The resulting spectral resolution is approximately $1.2 \AA$ (FWHM). The spectral setting covers the region from $6300 \AA<\lambda<9100 \AA$, with only a small spectral gap between the blue and red chips.

The data were reduced using the method presented by Simon \& Geha (2007), who modified the DEIMOS spec2d pipeline (Cooper et al. 2012; Newman et al. 2013) for stellar spectral analysis. The code was used to extract onedimensional, wavelength calibrated science spectra. The pipeline also outputs an associated sky spectrum for each slit. Radial velocities were measured by cross-correlation with a set of high $\mathrm{S} / \mathrm{N}$ templates measured with the same spectral setup, and the velocities were corrected for slit miscentering. We refer the reader to Simon \& Geha (2007) and Tollerud et al. (2012) for a more in-depth discussion of the radial velocity measurement technique.

\subsection{Photometry and Membership Selection}

We make use of previously published photometry for our spectroscopic analysis. The photometry is used in combination with isochrones to calculate initial temperature $\left(T_{\mathrm{eff}}\right)$ estimates, and fix surface gravities, $\log (g)$, for use in the spectroscopic analysis (see $\S 3$ ). We used the $M-T 2$ photometry for And I, And III, And V, And VII, and And X from the SPLASH survey (Beaton, comm), $R I_{C}$ photometry for NGC 147 and NGC 185 (Geha et al. 2010), V-i Subaru photometry for And II (Ho et al. 2012), and Megacam $g$ 'r' $i$ ' pho- 
tometry for M 32 (Howley et al. 2013). The inhomogeneity in the photometry does not affect the results of our analysis.

We rely on the membership selection presented by Tollerud et al. (2012), Ho et al. (2012), Geha et al. (2010), and Ho et al. (2014). The member stars were selected on the basis of radial velocity, position in a color-magnitude diagram, distance from the center of the dwarf galaxy, and dwarf/giant star spectral diagnostics including the strength of the $\mathrm{Na}$ I $\lambda 8190$ infrared doublet.

The analysis below assumes we have sampled the true abundance distribution of these galaxies in an unbiased way. Given the large luminosity of many of our galaxies, our spectroscopic member samples are limited to a small fraction, $<10 \%$, of all RGB member stars down to our limiting magnitude. Biases in the measured metallicity distribution could be injected if stringent color cuts were made, since metallicity and color are correlated. Our spectroscopic samples sample a wide range of colors, as seen in the CMDs shown by Tollerud et al. (2012). Furthermore, the majority of targets with colors discrepant from the visible RGBs are also classified as non-members based on their radial velocity. Another source of bias may be due to comparing different stellar populations, e.g. RGBs vs main sequence stars. Most chemical abundance studies of dwarf galaxies are based on the RGB population, and it is to these populations that we compare our sample throughout. In our analysis, we take into account our limited sampling of these galaxies, but caution the reader that minority populations in chemical abundance space may remain undetected.

\subsection{New And V and And X Masks}

We observed two new DEIMOS masks for And V and one for And X during an observing run on September 14-16, 2012, supplementing the previous DEIMOS observations of both galaxies by Tollerud et al. (2012). Objects with membership probability $P>0.05$ and $\mathrm{S} / \mathrm{N}<20 \AA^{-1}$ from Tollerud et al. (2012) were re-observed to provide higher S/N spectra. Each mask was observed between 1 and 4 hours.

We calculated membership probabilities for these new spectra using very similar criteria as for the other data. The probability based on radial velocity, $P_{v e l}$ was modeled as a Gaussian centered on the systemic velocity determined by Tollerud et al. (2012), and with $\sigma$ equal to the velocity dispersion measured in that work. The positional probability, $P_{p o s}$, was set to 1 within $r_{1 / 2}$ and modeled as a decreasing exponential elsewhere, using the half-light radius given in Table 1 We also calculated a probability based on position on a colormagnitude diagram. A star was given $P_{c m d}=1$ whenever its color fell between the $[\mathrm{Fe} / \mathrm{H}]=-2.5$ and $[\mathrm{Fe} / \mathrm{H}]=-0.5$ Yale isochrone $\mathrm{RGB}$ ridges (assuming $[\alpha / \mathrm{Fe}]=+0.4$ and $12 \mathrm{Gyr}$ ). If the color was blue-ward/red-ward of these color bounds, $P_{c m d}$ was down-weighted by the difference between the measured color and the blue/red ridge using a Gaussian with $\sigma=$ $\sigma_{\text {color }}$. Finally, we used the two dwarf-giant star discriminants, the Na I- $(V-I)$ spectral line diagnostic by Gilbert et al. (2006) and the Mg I- $\Sigma \mathrm{CaT}$ diagnostic by Battaglia \& Starkenburg (2012). These diagnostics only helped to confirm a few radial velocity non-members in our masks, so we did not incorporate them into our membership probability. The membership probability $P_{\text {memb }}$ for each star was then calculated as $P_{m e m b}=P_{v e l} \times P_{\text {pos }} \times P_{c m d}$. We use a low membership threshold of $P_{\text {memb }}=0.05$. We note that field contamination at the radial velocity of these two galaxies from MW and M 31 stars is expected to be small, and that our samples are concentrated mainly on the inner two half-light radii of both galaxies, where the contrast between member and non-member stars is highest.

\section{CHEMICAL ABUNDANCE ANALYSIS}

We measure the alpha to iron abundance ratio, $[\alpha / \mathrm{Fe}]$, in individual RGB spectra using the spectral synthesis technique described first by Kirby et al. (2008), expanded by Vargas et al. (2013) (V13). We describe the technique in $\S 3.1$ 3.2. Due to the distance to the M 31 system, our spectra have lower S/N than those analyzed by Kirby et al. (2008) or V13. We thus perform Monte Carlo tests to assess the recoverability of $[\alpha / \mathrm{Fe}]$, as described in $\$ 3.3$.

\subsection{Photometric Inputs and Synthetic Grid}

Our abundance technique measures iron and alpha abundances by finding the best-fit between each DEIMOS stellar spectrum and a synthetic grid of spectra. The grid has a fine sampling in $T_{\text {eff }}$, surface gravity $(\log (g))$, stellar metallicity, and $[\alpha / \mathrm{Fe}]_{\mathrm{atm}}$, where $[\alpha / \mathrm{Fe}]_{\mathrm{atm}}$ stands for the abundance ratio of alpha elements relative to iron, all varied in lockstep relative to their solar-scaled composition at a given metallicity. The metallicity represents the lockstep abundance variation of all elements other than $\mathrm{H}$ and $\mathrm{He}$, relative to their solar abundance. In practice, it is measured only from Fe lines. The full list of alpha elements is enumerated by Kirby (2011). In practice, only $\mathrm{Mg}, \mathrm{Si}, \mathrm{Ca}$, and $\mathrm{Ti}$ lines are used when fitting the spectra. When referring to the grid, we will use $[\alpha / \mathrm{Fe}]_{\mathrm{atm}}$, reserving unscripted $[\alpha / \mathrm{Fe}]$ for the "average" alpha abundance ratio, defined below.

Prior to the actual fitting, we determine $\log (g)$ from photometry. The alternate method of measuring $\log (g)$ from the spectra is not feasible due to a lack of measurable Fe II features needed for fixing ionization equilibrium. From the photometry, we also obtain an initial estimate of $T_{\text {eff }}$ which is then refined during the spectral fitting. We measure the photometric $T_{\text {eff }}$, and $\log (g)$ from the isochrone that passes closest to the reddening and extinction-corrected colors and magnitudes listed in $\S 2.2$, out of a grid of isochrones with varying metallicity from $[\mathrm{Fe} / \mathrm{H}]^{\text {iso }}=-2.5$ to solar, fixed age (12 Gyr), and $[\alpha / \mathrm{Fe}]^{\mathrm{iso}}(=+0.4)$. We calculate these values independently using the Yale-Yonsei (Kim et al. 2002), Dartmouth (Dotter et al. 2008), and PARSEC (Bressan et al. 2012) isochrone libraries. $[\alpha / \mathrm{Fe}]^{\text {iso }}=+0.4$ except for PAR$\mathrm{SEC}$, for which we use $[\alpha / \mathrm{Fe}]^{\text {iso }}=+0.0$ due to the present lack of $[\alpha / \mathrm{Fe}]^{\text {iso }}=+0.4$ isochrones. We measure the uncertainties in $T_{\text {eff }}$ and $\log (g)$ from 500 Monte Carlo resamplings of each star's color and magnitude, and using the standard deviation of the distribution of parameters. We average the results from the three independent isochrone libraries (using variance-weighted means).

Since our RGB sample may contain a wide spread of ages and alpha enhancements, we check whether using a different fixed age and $[\alpha / \mathrm{Fe}]^{\text {iso }}$ results in significant systematic shifts in $T_{\text {eff }}$ and $\log (g)$, which would then influence the abundance analysis. We test for the effect of changing $[\alpha / \mathrm{Fe}]^{\text {iso }}$ and age separately, using the Yale isochrone grid and recalculating $T_{\text {eff }}$ and $\log (g)$, for the stars in NGC 147, keeping the other parameters as before. If using $[\alpha / \mathrm{Fe}]^{\text {iso }}=+0.0, \log (g)$ shifts by only -0.01 dex, whereas the initial estimates of $T_{\text {eff }}$ shift by $-15 \mathrm{~K}$. If instead we use an age of $4 \mathrm{Gyr}, \log (g)$ changes by $0.16 \mathrm{dex}$, and the estimates of $T_{\text {eff }}$ by $-56 \mathrm{~K}$. The effect of 

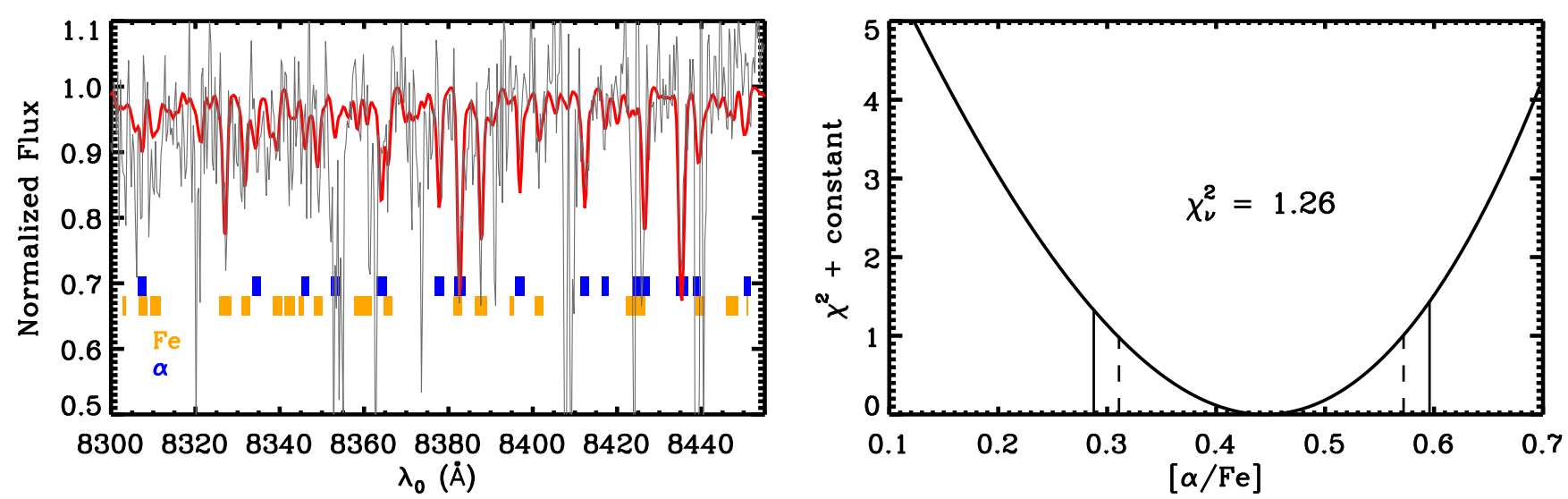

Figure 1. Left: Sample Keck/DEIMOS spectrum of a red giant branch star in And VII. The star has $[\mathrm{Fe} / \mathrm{H}]=-1.22$ and $[\alpha / \mathrm{Fe}]_{\text {atm }}=+0.44$. Only a small spectral range is shown for illustration purposes. Regions used for fitting Fe and alpha elements are shown in orange and blue, respectively. Right: Unreduced $\chi^{2}$ as a function of model $[\alpha / \mathrm{Fe}]_{\mathrm{atm}}$. The random uncertainty and full uncertainty (including the systemic error floor) are shown as dashed and solid lines, respectively. The reduced $\chi^{2}$ for the best-fitting model, $\chi_{\nu}^{2}$, is shown in the plot. The procedure for determining abundances and their uncertainties is described in Section 3.2

these systemic shifts, in particular $\log (g)$ which is not refined by the spectral analysis, will translate to systemic shifts in the abundances smaller than $0.05 \mathrm{dex}$, smaller the error floors in our measurements (see next subsection).

\subsection{Abundance Analysis}

After determining $\log (g)$ and the initial estimate of the $T_{\text {eff }}$ from isochrones, we measure the abundances using the same set of steps as V13. Prior to fitting, the science spectra are normalized, and the spectral resolution is refined as a function of wavelength. We use fits to sky emission lines extracted for each slit to measure the variation in resolution with wavelength, $\sigma_{s k y}(\lambda)$. We adjust $\sigma_{\text {sky }}$ with a constant rescaling factor for each spectral mask, which encodes the difference in slit imaging between the sky lines and the science spectra, i.e. $\sigma(\lambda)=k \times \sigma_{s k y}(\lambda)$. The factor $k$ is allowed to vary in a first pass of the abundance code (described below), and then fixed for each mask as the average $k$ for all stars in the mask. As in V13, we only fit spectral regions sensitive to Fe or alpha elements, excluding regions with significant telluric contamination (same regions as defined by Kirby et al. 2009) and a few strong lines such as the calcium triplet, which are not modeled adequately in the synthetic spectra. In addition, we now mask regions affected by strong sky emission. While sky lines are nominally subtracted by the pipeline, the subtraction is not perfect, an effect that is more important at low $\mathrm{S} / \mathrm{N}$. We measure the median sky flux in $500 \AA$-wide non-overlapping segments. We identify pixels where $f_{\text {sky }}(\lambda)>f_{\text {sky,median }}$, and $f_{\text {sky }}(\lambda)>0.25 \times f_{\text {star }}(\lambda)$, and mask them out when fitting the spectra.

We next fit each normalized spectrum against the aforementioned synthetic grid to find the best-fitting $[\mathrm{Fe} / \mathrm{H}]$ and $[\alpha / \mathrm{Fe}]_{\mathrm{atm}}$, while also allowing $T_{\text {eff }}$ to vary from the initial photometric estimate. The grid's $[\mathrm{Fe} / \mathrm{H}]$ and $[\alpha / \mathrm{Fe}]_{\mathrm{atm}}$ ranges are $-4.8 \leq[\mathrm{Fe} / \mathrm{H}] \leq 0.0$ and $-0.8 \leq[\alpha / \mathrm{Fe}]_{\text {atm }} \leq+1.2$, respectively, with a gradation of 0.1 dex in both parameters. In practice, $T_{\text {eff }}$ and $[\mathrm{Fe} / \mathrm{H}]$ are fit simultaneously, while $[\alpha / \mathrm{Fe}]_{\mathrm{atm}}$ is fit in a separate step. These steps are iterated to obtain convergence in all three parameters. During the fitting, we linearly interpolate the fluxes between the nearest spectra at each pixel wavelength. We only fit those spectral regions sensitive to changes in $\mathrm{Fe}$ or alpha element abundance (where alpha is one of $\mathrm{Mg}, \mathrm{Si}, \mathrm{Ca}$, or $\mathrm{Ti}$ ), and minimize the pixel-by-pixel flux variation between the synthetic models and the spectra using the Levenberg-Marquard minimization code mpfit.

The uncertainty in $[\mathrm{Fe} / \mathrm{H}]$ includes the covariance between $[\mathrm{Fe} / \mathrm{H}]$ and $T_{\text {eff }}$, resulting in a larger error than if either parameter was fit independently. For $[\alpha / \mathrm{Fe}]_{\mathrm{atm}}, \mathrm{V} 13$ found that a significant fraction of $\chi^{2}$ contours in the $\chi^{2}$ minimization were asymmetric, with a tendency for the $\chi^{2}$ contours flattening out towards lower $[\alpha / \mathrm{Fe}]_{\mathrm{atm}}$. Thus, they reported asymmetric $[\alpha / \mathrm{Fe}]_{\mathrm{atm}}$ error bars by finding the values of $[\alpha / \mathrm{Fe}]_{\mathrm{atm}}$ that satisfy $\chi^{2}=\chi_{\min }^{2}+1$, where $\chi_{\min }^{2}$ is the $\chi^{2}$ value for the best-fitting model. We adopt that procedure here and report both lower and upper $[\alpha / \mathrm{Fe}]_{\text {atm }}$ uncertainties, $\sigma^{-}$and $\sigma^{+}$. We note that in the few cases where the $\chi^{2}$ contour does not rise to $\chi_{\min }^{2}+1$, we adopt a lower uncertainty in $[\alpha / \mathrm{Fe}]_{\mathrm{atm}}$ equal to the entire range in $[\alpha / \mathrm{Fe}]_{\mathrm{atm}}$ between the best-fit value and the lower edge of the grid $\left([\alpha / \mathrm{Fe}]_{\mathrm{atm}}=-0.8\right)$. Finally, we add in quadrature an uncertainty floor to all $[\mathrm{Fe} / \mathrm{H}]$ $(\sigma=0.113)$ and $[\alpha / \mathrm{Fe}]_{\mathrm{atm}}(\sigma=0.082)$ measurements as in V13. The uncertainty floor reflects the difference between DEIMOS and literature high-resolution abundance measurements in the limit of high $\mathrm{S} / \mathrm{N}$ data (i.e., in the regime of small internal uncertainties). To illustrate the technique, we show in Figure 1 (left panel) a portion of a DEIMOS spectrum of an RGB in And VII, together with the best-fitting synthetic spectrum. The right panel shows the $\chi^{2}$ fit for $[\alpha / \mathrm{Fe}]_{\mathrm{atm}}$, as well as the uncertainties measured as described above.

We report the alpha abundance ratio, $[\alpha / \mathrm{Fe}]$, which we define as the arithmetic mean of $[\mathrm{Mg} / \mathrm{Fe}],[\mathrm{Si} / \mathrm{Fe}],[\mathrm{Ca} / \mathrm{Fe}]$, and $[\mathrm{Ti} / \mathrm{Fe}] 2$. We measure $[\alpha / \mathrm{Fe}]$ from the $[\alpha / \mathrm{Fe}]_{\mathrm{atm}}$ parameter fit by the abundance code, because the individual alpha elements are not measurable for most stars due to our low $\mathrm{S} / \mathrm{N}$. We calculate a correction term for converting $[\alpha / \mathrm{Fe}]_{\mathrm{atm}}$ to $[\alpha / \mathrm{Fe}]$, using DEIMOS spectra of Milky Way classical dSphs originally presented by Kirby et al. (2011). We have reanalyzed this sample in order to ensure maximum homogeneity between the Milky Way and M31 samples. The Milky Way dSph spectra have higher $\mathrm{S} / \mathrm{N}$ than our program sample, so that $[\alpha / \mathrm{Fe}]$ can be measured directly from the arithmetic mean of $[\mathrm{Mg} / \mathrm{Fe}],[\mathrm{Si} / \mathrm{Fe}],[\mathrm{Ca} / \mathrm{Fe}]$ and $[\mathrm{Ti} / \mathrm{Fe}]$ for a significant subset of spectra. We fit a third order polynomial to the differ-

\footnotetext{
2 Throughout the paper, we will simply refer to $[\alpha / \mathrm{Fe}]$ as the alpha abundance for a single star, reserving the bracket notation $\langle\ldots\rangle$ for averages over various population bins.
} 


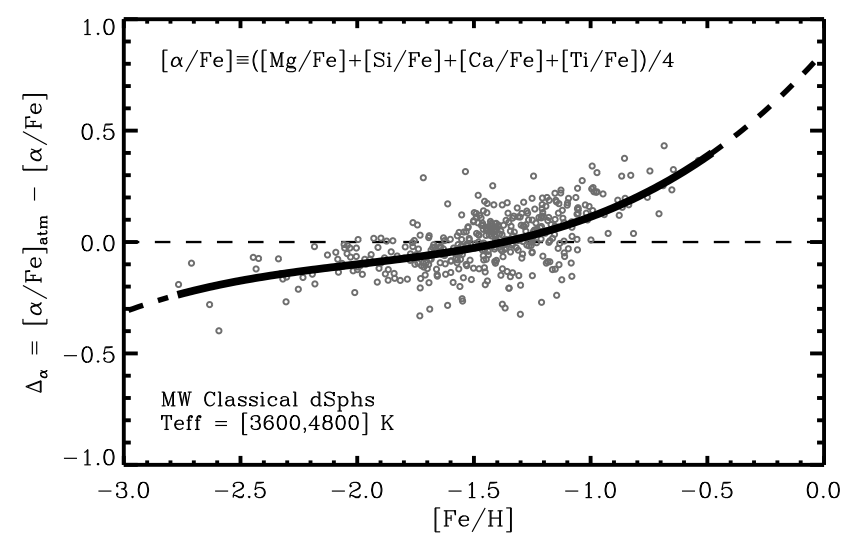

Figure 2. Difference between $[\alpha / \mathrm{Fe}]_{\text {atm }}$ and the arithmetic mean of the abundance ratios: $[\mathrm{Mg} / \mathrm{Fe}],[\mathrm{Si} / \mathrm{Fe}],[\mathrm{Ca} / \mathrm{Fe}]$, and $[\mathrm{Ti} / \mathrm{Fe}]$, plotted as a function of iron abundance, for RGB stars in six MW dSphs in the same range of $T_{\text {eff }}$ as our program stars. The mean is denoted as $[\alpha / \mathrm{Fe}]$ throughout the paper. The solid line is a third-order polynomial fit to the difference $\Delta \equiv[\alpha / \mathrm{Fe}]_{\mathrm{atm}}-[\alpha / \mathrm{Fe}]$, between $-2.8<[\mathrm{Fe} / \mathrm{H}]<-0.5$, using the comparison MW dSph stars. We apply this correction to $[\alpha / \mathrm{Fe}]_{\text {atm }}$ to calculate $[\alpha / \mathrm{Fe}]$ for our sample. See $\$ 3.2$ for more details. The dotted extensions are an extrapolation based on the polynomial describing the solid line. Unless stated otherwise, we do not consider the $[\alpha / \mathrm{Fe}]$ values in the extrapolated region in our analysis.

ence between $[\alpha / \mathrm{Fe}]_{\mathrm{atm}}$ and $[\alpha / \mathrm{Fe}]$ as a function of $[\mathrm{Fe} / \mathrm{H}]$, further restricting the MW dSph sample only to stars in the $T_{\text {eff }}$ range of our M 31 dwarf galaxy sample, and for which all four abundance ratios were measured.

Figure 2$]$ shows the difference $[\alpha / \mathrm{Fe}]_{\mathrm{atm}}-[\alpha / \mathrm{Fe}]$. The relation between $[\alpha / \mathrm{Fe}]_{\mathrm{atm}}$ and $[\alpha / \mathrm{Fe}]$ varies with $[\mathrm{Fe} / \mathrm{H}]$ at least in our $T_{\text {eff }}$ range. We note that $[\alpha / \mathrm{Fe}]_{\mathrm{atm}}$, which is fit from all lines of $\mathrm{Mg}, \mathrm{Si}, \mathrm{Ca}$, and $\mathrm{Ti}$, need not be equal to $[\alpha / \mathrm{Fe}]$, since the relative strength and number of different spectral lines and different elements varies with $T_{\text {eff }}$ and metallicity. We thus favor the definition of $[\alpha / \mathrm{Fe}]$ which allows easier comparison to other literature results. We also note that without the correction term, the $[\alpha / \mathrm{Fe}]$ measurements for the Fornax dSph would appear significantly enhanced, in contrast to what is measured from the individual alpha elements by our reanalysis of MW dSph data, by Kirby et al. (2011), and by Letarte et al. (2010), the latter based on $\mathrm{R} \sim 20,000$ spectra.

To determine $[\alpha / \mathrm{Fe}]$, we apply the correction function discussed above (black line in Figure 2) to all $[\alpha / \mathrm{Fe}]_{\text {atm }}$ measurements, including those in the comparison Milky Way $\mathrm{dSph}$ sample. We verified that the vertical scatter about the best-fit line is consistent with the data uncertainty. We thus keep $\sigma_{[\alpha / \mathrm{Fe}]}=\sigma_{[\alpha / \mathrm{Fe}]_{\mathrm{atm}}}$. Due to a lack of calibrators with $[\mathrm{Fe} / \mathrm{H}]>-0.5$, the correction term for this metallicity range is only an extrapolation. Hence, we restrict our analysis to those stars with $[\mathrm{Fe} / \mathrm{H}]<-0.5$, except where noted. Using a more metal-poor sample, V13 determined a positive correction of +0.063 was needed for $[\alpha / \mathrm{Fe}]_{\mathrm{atm}}$ to equal $[\alpha / \mathrm{Fe}]$. However, this comparison was done on the basis of only metal-poor stars with a higher average $T_{\text {eff. }}$. Using the Milky Way dSphs sample, we confirm that the difference between $[\alpha / \mathrm{Fe}]_{\mathrm{atm}}$ and $[\alpha / \mathrm{Fe}]$ is smaller for higher $T_{\text {eff }}$ stars. The $[\mathrm{Fe} / \mathrm{H}]$ and $[\alpha / \mathrm{Fe}]$ measurements are converted from the Anders \& Grevesse (1989) abundance scale to the newer Asplund et al. (2009) scale, as in V13.

\subsection{Precision of $[\alpha / \mathrm{Fe}]$ at low $S / N$ : Synthetic Spectral Tests}

The distance to the M31 dwarf galaxies limits the S/N obtainable with reasonable observing times. The maximum $\mathrm{S} / \mathrm{N}$ in our sample is only $25 \AA^{-1}$. In contrast, the sample from which we derived the correction between $[\alpha / \mathrm{Fe}]_{\mathrm{atm}}$ and $[\alpha / \mathrm{Fe}]$ have a median S/N between $42 \AA^{-1}$ (Fornax) and 150 $\AA^{-1}$ (Draco). While $\chi^{2}$ provides an easily measurable estimate of the uncertainty, it is plausible to suspect there is a bias in the measurement of $[\alpha / \mathrm{Fe}]$ where lower $[\alpha / \mathrm{Fe}]$ values would be harder to observe due to the lower $\mathrm{S} / \mathrm{N}$ of the line at a fixed spectral $\mathrm{S} / \mathrm{N}$. To assess the precision of our analysis pipeline, we turn to Monte Carlo simulations.

We create continuum-normalized mock spectra using the synthetic spectral library from Kirby (2011). We convolve the spectra with a Gaussian kernel with FWHM $=1.2 \AA$, so as to closely reproduce the DEIMOS spectral resolution, and bin the degraded spectrum to the DEIMOS pixel size and spectral range. To roughly simulate the variation of $\mathrm{S} / \mathrm{N}$ with wavelength, we add a pseudo-continuum derived from a spline fit to a DEIMOS template of a cool RGB star. The mock spectra have $T_{\text {eff }}=4,200 \mathrm{~K}$, metallicities ranging from $[\mathrm{Fe} / \mathrm{H}]=-4.0$ to $[\mathrm{Fe} / \mathrm{H}]=-0.5$ (in steps of $0.5 \mathrm{dex}$ ) and $[\alpha / \mathrm{Fe}]=[+0.0,+0.4]$. The $T_{\text {eff }}$ value represents the average $T_{\text {eff }}$ in our sample. For each parameter combination, $\log (g)$ value is set by comparison to a grid of Dartmouth isochrones, and finding the $\log (g)$ corresponding to the appropriate $T_{\text {eff }},[\mathrm{Fe} / \mathrm{H}]$, and $[\alpha / \mathrm{Fe}]$. For $[\mathrm{Fe} / \mathrm{H}] \leq-2.5$, we use the $[\mathrm{Fe} / \mathrm{H}]=-2.5$ isochrones, noting that the differences in isochrone RGBs decrease towards lower metallicities. We then add Gaussian noise to each pixel consistent with its flux uncertainty (updating the variance array) such that the average $\mathrm{S} / \mathrm{N}$ in the $\mathrm{CaT}$ region is one of $\mathrm{S} / \mathrm{N}=[8,15,30] \AA^{-1}$. We create 200 mock realizations for each set of parameters at each $\mathrm{S} / \mathrm{N}$, and analyze the spectrum with our abundance pipeline, using as initial inputs the true $T_{\text {eff }}$ and $\log (g)$ of the synthetic spectrum.

Figure 3 shows the scatter in $[\alpha / \mathrm{Fe}]$ against $[\mathrm{Fe} / \mathrm{H}]$ for the case of a $T_{\text {eff }}=4200 \mathrm{~K},[\mathrm{Fe} / \mathrm{H}]=[-2.5,-1.5,-0.5]$, $[\alpha / \mathrm{Fe}]=[+0.0,+0.4]$ spectra, showing that for $\mathrm{S} / \mathrm{N} \gtrsim 15 \AA^{-1}$, we can distinguish between a population of $[\alpha / \mathrm{Fe}]=+0.0$ and $[\alpha / \mathrm{Fe}]=+0.4$ objects. At $\mathrm{S} / \mathrm{N}<15 \AA^{-1}$, the MC tests suggest we can still recover the average $[\alpha / \mathrm{Fe}]$ of a population, albeit with a significant increase in the random scatter. Because of this, we choose $\mathrm{S} / \mathrm{N}=15 \AA^{-1}$ as our lower $\mathrm{S} / \mathrm{N}$ cut for $[\alpha / \mathrm{Fe}]$. The same set of $\mathrm{MC}$ tests show that $[\mathrm{Fe} / \mathrm{H}]$ can be recovered accurately for $\mathrm{S} / \mathrm{N}>8 \AA^{-1}$. For all analyses involving $[\alpha / \mathrm{Fe}]$ we apply the threshold $\mathrm{S} / \mathrm{N}>15 \AA^{-1}$; we use the lower threshold for $[\mathrm{Fe} / \mathrm{H}]\left(\mathrm{S} / \mathrm{N}>8 \AA^{-1}\right)$ only to calculate average metallicities and radial metallicity trends.

\section{RESULTS}

The $[\alpha / \mathrm{Fe}]$ abundance ratio is an indicator of the star formation timescale in a galaxy. Qualitatively, high average alpha abundances can be explained as a being due to short star formation timescales, whereas low average alpha abundances are associated with slower star formation timescales. We have measured both alpha and iron abundances in nine dwarf galaxies of M 31, sampling a large range in V-band absolute magnitude from $\mathrm{M}_{V} \sim-7.4$ (And X) to $\mathrm{M}_{V} \sim-16.4$ (M 32). Figure 4 presents our full dataset, plotted in the $[\alpha / \mathrm{Fe}]-[\mathrm{Fe} / \mathrm{H}]$ abundance plane.

A visual inspection of the data shows a variety of abundance patterns in $[\alpha / \mathrm{Fe}]-[\mathrm{Fe} / \mathrm{H}]$ space between the different dwarf galaxies. The plot also indicates the presence of signif- 


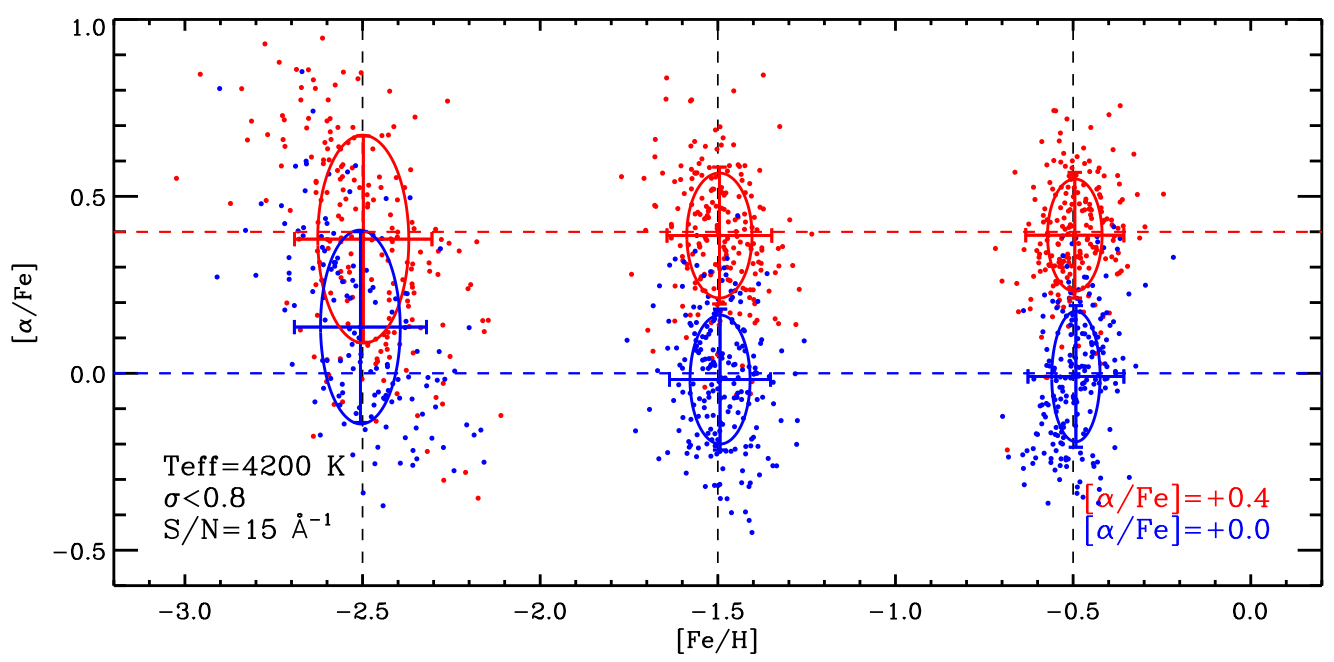

Figure 3. Distribution of recovered $[\alpha / \mathrm{Fe}]$ and $[\mathrm{Fe} / \mathrm{H}]$ measurements at low $\mathrm{S} / \mathrm{N}$ using mock synthetic spectra. We created mock spectra for the following parameter combinations: $[\mathrm{Fe} / \mathrm{H}]=[-2.5,-1.5,-0.5]$, and $[\alpha / \mathrm{Fe}]=[+0.0,+0.4]$. For each parameter set, we draw from our synthetic spectral library $($ degraded to DEIMOS resolution wavelength coverage, and desired S/N) to create 200 mock spectra, which we then process through our abundance pipeline. The ellipses show the $1 \sigma$ uncertainties. We thus can distinguish between an "alpha-enriched" (+0.4) population and a "alpha-solar" (+0.0) population at this low S/N, although the latter will be slightly biased at $[\mathrm{Fe} / \mathrm{H}]<-2.5$. We restrict our $[\alpha / \mathrm{Fe}]$ measurements to $\mathrm{S} / \mathrm{N}>15 \AA^{-1}$ spectra.

Table 2

Iron and Alpha Abundance Results

\begin{tabular}{lccccc}
\hline \hline Galaxy & $\begin{array}{c}\text { R.A. } \\
\text { (J2000) }\end{array}$ & $\begin{array}{c}\text { Decl. } \\
\text { (J2000 })\end{array}$ & {$[\mathrm{Fe} / \mathrm{H}]$} & {$[\alpha / \mathrm{Fe}]$} & Flag \\
\hline M 32 & $00: 42: 39.32$ & $+40: 53: 27.0$ & $-0.79 \pm 0.13$ & $+0.01_{-0.31}^{+0.30}$ & \\
M 32 & $00: 42: 42.30$ & $+40: 54: 39.6$ & $-1.15 \pm 0.13$ & $+0.81_{-0.22}^{+0.20}$ & \\
M 32 & $00: 42: 53.34$ & $+40: 51: 35.6$ & $-0.98 \pm 0.13$ & $+0.46_{-0.30}^{+0.26}$ & \\
NGC 185 & $00: 38: 09.18$ & $+48: 13: 45.6$ & $-1.61 \pm 0.17$ & $+0.52_{-0.22}^{+0.20}$ & \\
NGC 185 & $00: 38: 12.51$ & $+48: 13: 09.2$ & $-1.91 \pm 0.18$ & $-0.18_{-0.62}^{+0.42}$ & u \\
NGC 185 & $00: 38: 22.49$ & $+48: 09: 47.6$ & $-1.49 \pm 0.16$ & $+0.74_{-0.20}^{+0.18}$ & \\
NGC 185 & $00: 38: 22.88$ & $+48: 15: 47.2$ & $-0.99 \pm 0.13$ & $+0.32_{-0.23}^{+0.22}$ & \\
NGC 185 & $00: 38: 23.46$ & $+48: 11: 07.9$ & $-0.83 \pm 0.13$ & $-0.10_{-0.48}^{+0.31}$ & \\
NGC 185 & $00: 38: 24.95$ & $+48: 21: 54.3$ & $-0.82 \pm 0.13$ & $+0.15_{-0.20}^{+0.20}$ & \\
NGC 185 & $00: 38: 25.10$ & $+48: 12: 52.0$ & $-0.68 \pm 0.12$ & $-0.10_{-0.23}^{+0.23}$ & \\
& & & & & \\
\hline
\end{tabular}

Note. - Table 2 is published in its entirety in the electronic edition of the Astrophysical Journal. A portion is shown here for guidance regarding its form and content. Flags on last column: (1) u - Lower limit uncertainty in $[\alpha / \mathrm{Fe}]$ is outside of our grid, thus we set $\sigma_{[\alpha / \mathrm{Fe}]}^{-}=[\alpha / \mathrm{Fe}]-\min \left([\alpha / \mathrm{Fe}]_{\text {grid }}\right)=$ $[\alpha / \mathrm{Fe}]-(-0.8) .(2) \mathrm{mr}=[\mathrm{Fe} / \mathrm{H}]>-0.5$ star. (3) $\mathrm{f}:$ Only $[\mathrm{Fe} / \mathrm{H}]$ is available for this star

icant iron abundance spreads in all dwarf galaxies in the sample, confirming previous results based on photometric metallicities (e.g., Kalirai et al.|2010), and measured directly from the calcium triplet (Ho et al. 2014). This is the first time that iron abundance spreads are measured directly from iron lines in M31 satellite galaxies. The sequence of panels also illustrates qualitatively the correlation between luminosity and average iron abundance (Kirby et al. 2013). NGC 147 appears to scatter above the relation. Interestingly, this galaxy has been shown to have tidal features in PanDAS photometry (McConnachie et al. 2009). It is thus possible it was more luminous in the past. If so, this could explain its relatively high metallicity given its present day luminosity.

\subsection{Mean Chemical Abundance Trends}

Average chemical trends, e.g., the luminosity-metallicity relation, are useful in constraining the properties of galaxy evolution as a function of luminosity or other global parameters. We thus quantify the average (characteristic) alpha and iron chemical properties, $\langle[\alpha / \mathrm{Fe}]\rangle$ and $\langle[\mathrm{Fe} / \mathrm{H}]\rangle$, for each dwarf galaxy. We calculate $\langle[\alpha / \mathrm{Fe}]\rangle$ using spectra with $\mathrm{S} / \mathrm{N}>15 \AA^{-1}$ and $[\mathrm{Fe} / \mathrm{H}]<-0.5$. We exclude the stars with $[\mathrm{Fe} / \mathrm{H}]>-0.5$ because we do not have a calibrating sample that allows us to verify the relation between $[\alpha / \mathrm{Fe}]_{\mathrm{atm}}$ and $[\alpha / \mathrm{Fe}]$ (see $\S 3.2$ ), but discuss the effect of excluding these stars in particular galaxies.

We calculate the mean iron abundance, $\langle[\mathrm{Fe} / \mathrm{H}]\rangle$, using all spectra with $\mathrm{S} / \mathrm{N}>8 \AA^{-1}$. We use this lower $\mathrm{S} / \mathrm{N}$ cut because the number of iron-sensitive spectral regions is larger than for $[\alpha / \mathrm{Fe}]_{\mathrm{atm}}$, so that even lower $\mathrm{S} / \mathrm{N}$ are useful for iron abundance measurements. Using this larger sample serves a twofold purpose. It allows us to provide a better constraint on $\langle[\mathrm{Fe} / \mathrm{H}]\rangle$ and to check whether small number statistics affect our $[\alpha / \mathrm{Fe}]$ samples. To do the latter, we compare the $[\mathrm{Fe} / \mathrm{H}]$ distribution for the alpha sample to the $[\mathrm{Fe} / \mathrm{H}]$ distribution for the full $[\mathrm{Fe} / \mathrm{H}]$ sample for each galaxy. With the exception of M 32 the distributions agree. For M 32, the five stars with $[\alpha / \mathrm{Fe}]$ are clustered at $[\mathrm{Fe} / \mathrm{H}]>-1.5$ whereas the $[\mathrm{Fe} / \mathrm{H}]$ measurements $\operatorname{span}-3<[\mathrm{Fe} / \mathrm{H}]<0$. This suggests that the average $\langle[\alpha / \mathrm{Fe}]\rangle$ for the M 32 sample is not representative of this galaxy's alpha enhancement, and do not consider it further in this subsection 3 .

We assess the uncertainty in $\langle[\mathrm{Fe} / \mathrm{H}]\rangle$ and $\langle[\alpha / \mathrm{Fe}]\rangle$ from the dispersion in the data using the Student $t$-distribution, thus taking into account the small sample sizes in various of our galaxies. We measure $68 \%$ confidence intervals for both $\langle[\mathrm{Fe} / \mathrm{H}]\rangle$ and $\langle[\alpha / \mathrm{Fe}]\rangle$ dividing the confidence interval for a single measurement by $N^{1 / 2}$ (standard error of the mean). In addition, we set an uncertainty floor on the means equal to the uncertainty floors described in $\S 3.2$. We tabulate our measurements of $\langle[\alpha / \mathrm{Fe}]\rangle$ and $\langle[\mathrm{Fe} / \mathrm{H}]\rangle$ in Table 3

The bottom-right panel of Figure 4 shows $\langle[\alpha / \mathrm{Fe}]\rangle$ versus $\langle[\mathrm{Fe} / \mathrm{H}]\rangle$. For this panel only, we have plotted $\langle[\mathrm{Fe} / \mathrm{H}]\rangle$ as measured from $\mathrm{S} / \mathrm{N}>15 \AA^{-1}$, so that both $\langle[\alpha / \mathrm{Fe}]\rangle$

${ }^{3}$ We do use the M 32's data later when comparing $\langle[\alpha / \mathrm{Fe}]\rangle$ at fixed metallicity. 


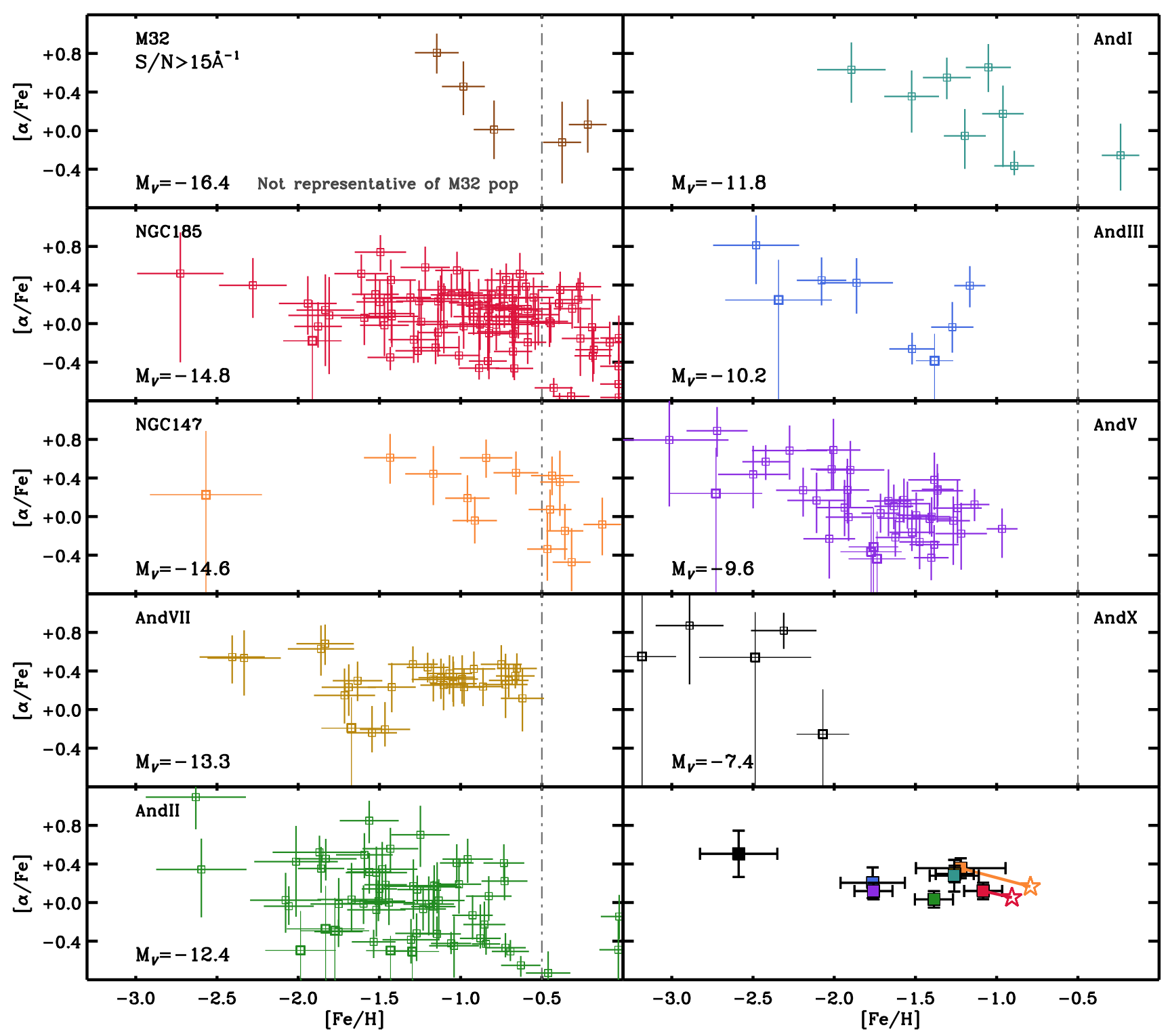

Figure 4. $[\alpha / \mathrm{Fe}]$ as a function of $[\mathrm{Fe} / \mathrm{H}]$ for our entire dataset. The dwarf galaxies are shown from most to least luminous in $\mathrm{V}$-band (top to bottom). The same color scheme is used to denote each galaxy in all following plots. The dash-dotted line at $[\mathrm{Fe} / \mathrm{H}]=-0.5$ indicates that $[\alpha / \mathrm{Fe}]$ values for higher metallicities are uncertain, due to the lack of calibrators to test the relation between $[\alpha / \mathrm{Fe}]_{\mathrm{atm}}$ and $\langle[\alpha / \mathrm{Fe}]\rangle$ (see $\S 3.2$ for discussion). Each square is a measurement of $[\alpha / \mathrm{Fe}]$ using only spectra with $\mathrm{S} / \mathrm{N} \geq 15 \AA^{-1}$. Bottom-right panel: We plot the average alpha abundance, $\langle[\alpha / \mathrm{Fe}]\rangle$, using $[\mathrm{Fe} / \mathrm{H}]<-0.5$ stars against the mean metallicity, $\langle[\mathrm{Fe} / \mathrm{H}]\rangle$, calculated from the same stars. For NGC 185 and NGC 147, we include a star symbol indicating the change in $\langle[\alpha / \mathrm{Fe}]\rangle$ and $\langle[\mathrm{Fe} / \mathrm{H}]\rangle$ if the $[\mathrm{Fe} / \mathrm{H}]>-0.5$ stars are included. We do not include M 32 in this panel because the $[\alpha / \mathrm{Fe}]$ sample is not representative of the galaxy's population $(\S 4.1]$.

and $\langle[\mathrm{Fe} / \mathrm{H}]\rangle$ are measured from the same sample. If the $[\mathrm{Fe} / \mathrm{H}]>-0.5$ stars are included, $\langle[\alpha / \mathrm{Fe}]\rangle$ decreases by -0.19 dex (NGC 147) and -0.07 dex (NGC 185), as shown by the star symbols. Given $\langle[\alpha / \mathrm{Fe}]\rangle=+0.36 \pm 0.11$ and $\langle[\alpha / \mathrm{Fe}]\rangle=+0.12 \pm 0.09$ for NGC 147 and NGC 185 , respectively, the changes resulting from ignoring the $[\mathrm{Fe} / \mathrm{H}]>-0.5$ stars cause some tension in $\langle[\alpha / \mathrm{Fe}]\rangle$ only in the case of NGC 147.

The bright dwarf galaxies NGC 185, and NGC 147 appear to have a small fraction of stars with $[\mathrm{Fe} / \mathrm{H}]>-0.5$. Since our synthetic spectral grid does not extend to $[\mathrm{Fe} / \mathrm{H}]>0$, observed stars at those metallicities will be best-fit by a $[\mathrm{Fe} / \mathrm{H}]=0$ spectrum. The number of such stars is low, ranging from $13 / 312$ in NGC 185 to $31 / 205$ in NGC 147. Based on the results of a CaT study of the same DEIMOS sample by Ho et al. (2014), excluding these stars would modify $\langle[\mathrm{Fe} / \mathrm{H}]\rangle$ by less than 0.15 dex.

We plot in Figure 5 the resulting average alpha abundance for each galaxy against galaxy luminosity, distance from M31, global kinematics, and surface brightness. We also include data for six MW dwarf galaxies (Fornax, Leo I, Leo II, Draco, Sextans, and Ursa Minor) reanalyzed with our code for purposes of comparison and reference (see $\$ 3.2$ ). We caution, however, that the homogeneous sample of MW satellites is not complete in our magnitude range. Thus we avoid comparing the MW and M31 satellites as populations. We defer such comparison to $\$ 4.2$ after adding other bright MW satellites from literature data, not included here as their sampling 

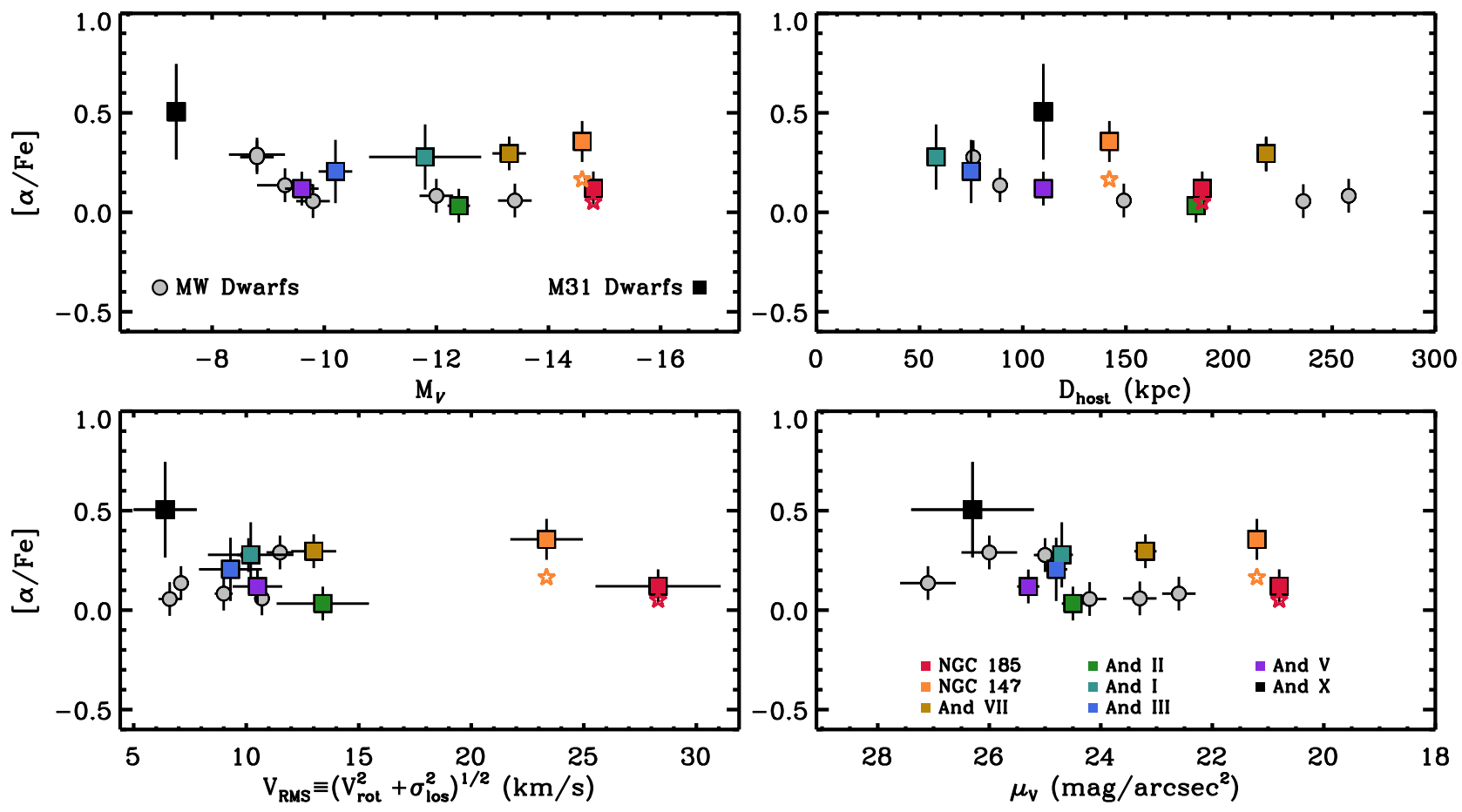

Figure 5. Mean alpha abundance, $\langle[\alpha / \mathrm{Fe}]\rangle$, in each of the nine dwarf galaxies as a function of (a) luminosity, (b) distance from host galaxy (either M 31 or Milky Way) (c) $\mathrm{V}_{r m s}$, the quadrature sum of line-of-sight velocity dispersion and rotation, and (d) central surface brightness. As discussed in $\S 4.1$ we only use stars with $[\mathrm{Fe} / \mathrm{H}]<-0.5$. For NGC 147 and NGC 185 , we plot a star symbol for the average alpha abundance calculated when including $[\mathrm{Fe} / \mathrm{H}]>-0.5$ stars. The change is non-negligible only for NGC 147. The $1 \sigma$ error in $\langle[\alpha / \mathrm{Fe}]\rangle$ takes into account the variation in sample size for each galaxy. The X-axis values and $1 \sigma$ uncertainties for each of the four properties plotted are taken from the literature, and summarized in Table 1 The M 31 dwarf galaxies are color-coded as shown in the bottom right panel (same as in Figure 4. We compare the sample against DEIMOS data for six classical MW dSphs analyzed homogeneously $(\$ 3.2$, shown as gray circles.

may not be appropriate for calculating galaxy-wide average chemical properties.

\subsection{1. $\langle[\alpha / \mathrm{Fe}]\rangle$ vs Luminosity}

We compare the chemical properties as a function of galaxy luminosity (Figure 5 top-left panel). The average alpha abundance ratios of $\mathrm{M} 31$ dwarf galaxies fainter than $\mathbf{M}_{V}=-10$ (i.e., And X, And III, and And V) are indistinguishable from the Milky Way dwarf galaxies of comparable luminosity (Leo II, Draco, Sextans and Ursa Minor). In particular, And V and And III have intermediate $\langle[\alpha / \mathrm{Fe}]\rangle$ values, consistent with those of Leo II and Sextans at almost the same luminosity. At higher galaxy luminosities, there is a clear distinction between the alpha enhancements of And VII $(\langle[\alpha / \mathrm{Fe}]\rangle \sim+0.3)$ and And II $(\langle[\alpha / \mathrm{Fe}]\rangle \sim+0.0)$. At similar luminosities, both MW dwarf galaxies in our comparison sample (Fornax and Leo I) have $\langle[\alpha / \mathrm{Fe}]\rangle$ close to And II (between $\sim+0.0$ and +0.1$)$. The MW and M 31 samples partially overlap in their luminosity range; however, the M31 sample contains more luminous dwarf galaxies. Out of the three brightest galaxies in our sample, we again find a range in alpha abundances, with NGC 147 having higher $\langle[\alpha / \mathrm{Fe}]\rangle$ than NGC 185 by $\sim 0.3$ (although the value is indistinguishable from NGC 147 if the $[\mathrm{Fe} / \mathrm{H}]>-0.5$ stars are included). In summary, we find that the M 31 dwarf satellites have a range of average alpha abundances enhancements, and there is no evidence for this trend being correlated with galaxy luminosity, at least for $\mathrm{M}_{V}<-9$.

\subsection{2. $\langle[\alpha / \mathrm{Fe}]\rangle$ vs Distance from Host}

We explore whether the environment plays a role in driving the variation in chemical properties by plotting the average alpha abundance against galactocentric distance from M 31 (top-right panel of Figure 5). For reference, the plot also includes the same set of comparison Milky Way dSphs, plotted against galactocentric distance from the Milky Way. We do not measure a significant correlation with environment, even at a host galaxy separation as large as that of And VII, the most distant M 31 satellite in our sample. If star formation in these dwarfs were very strongly influenced by environment, we might expect the objects closer to M31 to show signs of truncated star formation histories, and thus higher $\langle[\alpha / \mathrm{Fe}]\rangle$. However, one of the most alpha-enhanced systems is also one of the most distant (And VII). A full analysis of the role of environment requires knowledge of the orbital history of each satellite, which is not currently available.

\subsection{3. $\langle[\alpha / \mathrm{Fe}]\rangle$ vs Kinematics}

We next compare the average alpha abundances against global galaxy kinematics (bottom-left panel of Figure 5). We plot $\langle[\alpha / \mathrm{Fe}]\rangle$ against $v_{r m s}$, defined as the quadrature sum of $\sigma_{l o s}$ and the maximum rotation velocity $v_{\text {rot }}$, for those galaxies that show significant rotation, and equal to $\sigma_{l o s}$ otherwise. We use the spatially averaged (not central) $\sigma_{l o s}$, for NGC 185 and NGC 147, since a significant fraction of those data extend beyond their respective half-light radius. Taking into account both rotation and dispersion, there is no clear trend of alpha abundances with kinematics. Claims of such correlations have 
Table 3

M31 Dwarf Galaxy Average Chemical Properties

\begin{tabular}{|c|c|c|c|c|c|c|c|c|c|c|}
\hline Galaxy & $\langle[\mathrm{Fe} / \mathrm{H}]\rangle^{a}$ & $\mathrm{~N}$ & $\begin{array}{l}\langle[\alpha / \mathrm{Fe}]\rangle^{b} \\
{[\mathrm{Fe} / \mathrm{H}]<-0.5}\end{array}$ & 5 & $\begin{array}{c}\langle[\alpha / \mathrm{Fe}]\rangle^{b} \\
{[\mathrm{Fe} / \mathrm{H}]=[-2.5,}\end{array}$ & $\begin{array}{c}\mathrm{N} \\
1.5]\end{array}$ & $\begin{array}{c}\langle[\alpha / \mathrm{Fe}]\rangle^{b} \\
{[\mathrm{Fe} / \mathrm{H}]=[-2.0,-1}\end{array}$ & $\begin{array}{l}\mathrm{N} \\
1.0]\end{array}$ & $\begin{array}{c}\langle[\alpha / \mathrm{Fe}]\rangle^{b} \\
{[\mathrm{Fe} / \mathrm{H}]=[-1.5,-0}\end{array}$ & $\begin{array}{r}\mathrm{N} \\
0.5]\end{array}$ \\
\hline M 32 & $-1.153 \pm 0.165$ & $32^{c}$ & $0.425 \pm 0.319$ & $3^{d}$ & & & & & $0.425 \pm$ & 3 \\
\hline NGC 185 & $-0.917 \pm 0.117$ & $299^{c}$ & $0.120 \pm 0.085$ & 71 & $0.167 \pm 0.085$ & 9 & $0.137 \pm 0.085$ & 31 & $0.107 \pm 0.085$ & 61 \\
\hline NGC 147 & $-0.491 \pm 0.117$ & $184^{c}$ & $0.356 \pm 0.103$ & 7 & & & & & $0.378 \pm 0.121$ & 6 \\
\hline And VII & $-1.240 \pm 0.117$ & 90 & $0.296 \pm 0.085$ & 29 & $0.293 \pm 0.126$ & 9 & $0.257 \pm 0.085$ & 17 & $0.297 \pm 0.085$ & 20 \\
\hline And II & $-1.365 \pm 0.117$ & 248 & $0.033 \pm 0.085$ & 56 & $0.096 \pm 0.085$ & 21 & $0.041 \pm 0.085$ & 40 & $-0.049 \pm 0.085$ & 33 \\
\hline And I & $-1.109 \pm 0.117$ & 31 & $0.278 \pm 0.164$ & 7 & & & $0.427 \pm 0.157$ & 5 & $0.192 \pm 0.225$ & 5 \\
\hline And III & $-1.808 \pm 0.117$ & 35 & $0.205 \pm 0.159$ & 8 & $0.333 \pm 0.208$ & 5 & $0.026 \pm 0.197$ & 5 & $-0.009 \pm 0.312$ & 3 \\
\hline And V & $-1.712 \pm 0.117$ & 80 & $0.119 \pm 0.085$ & 40 & $0.131 \pm 0.085$ & 23 & $-0.004 \pm 0.085$ & 28 & $-0.014 \pm 0.085$ & 14 \\
\hline And $\mathrm{X}$ & $-2.298 \pm 0.123$ & 16 & $0.505 \pm 0.240$ & $5^{e}$ & $0.368 \pm 0.446$ & 3 & & & & \\
\hline
\end{tabular}

a $\langle[\mathrm{Fe} / \mathrm{H}]\rangle$ calculated from spectra with $\mathrm{S} / \mathrm{N}>8 \AA^{-1}$.

$\mathrm{b}\langle[\alpha / \mathrm{Fe}]\rangle$ calculated from spectra with $\mathrm{S} / \mathrm{N}>15 \AA^{-1}$, for the metallicity ranges specified below.

$\mathrm{c}\langle[\mathrm{Fe} / \mathrm{H}]\rangle$ for M 32, NGC 185 and NGC 147 may be biased (low) estimates, as there may be [Fe/H] $>0$ stars not measurable by our grid (see $\$ 3.2$.

$\mathrm{d}\langle[\alpha / \mathrm{Fe}]\rangle$ for $\mathrm{M} 32$ is not representative of this galaxy's population.

$\mathrm{e}\langle[\alpha / \mathrm{Fe}]\rangle$ for And $\mathrm{X}$ is possibly a biased (high) estimate, since 3 of 5 stars have large $\sigma^{-}$uncertainties in [ $\left.\alpha / \mathrm{Fe}\right]$ (see Figure 4 ).

been long reported in extra-galactic studies of giant ellipticals (e.g. Trager et al.2000; Thomas et al. 2005; Conroy et al. 2014; Greene et al. 2013). The lack of kinematic-abundance correlation suggests a difference in the formation mechanisms of giant versus dwarf elliptical (and spheroidal) systems.

\subsection{4. $\langle[\alpha / \mathrm{Fe}]\rangle$ vs Stellar Density}

In the bottom-right panel of Figure 5 we compare the average alpha abundance against the central surface brightness, $\mu_{V}$, a proxy for stellar mass density, since it could be expected that dwarf galaxies with higher stellar density could form stars more efficiently (Revaz \& Jablonka 2012). We do not detect any clear correlation. However, we note that our sample's radial coverage precludes a direct comparison at similar radii for all galaxies. The quoted $\mu_{V}$ only encapsulates the density in the central region, whereas a significant fraction of our stellar sample is beyond the inner half-light radius.

\subsection{Alpha Abundance Trends with Metallicity}

The distribution of $[\alpha / \mathrm{Fe}]$ as a function of $[\mathrm{Fe} / \mathrm{H}]$ reflects the chemical enrichment pathway within a galaxy. Roughly, the ISM evolves from metal-poor and alpha-enhanced towards higher metallicities and lower alpha abundances. While this picture is likely overly simplified for complex stellar populations, it is a good first step in finding differences between galaxies. Figure 4 hints at significant differences between the various galaxies in our sample. And $\mathrm{V}$ is the most salient example for an internal trend of decreasing alpha abundance with rising iron abundance, and for a bulk population with $[\alpha / \mathrm{Fe}] \lesssim 0.0$. In contrast, And VII is distinguished by a lack of a trend towards lower alphas: the majority of And VII stars in our sample have $[\alpha / \mathrm{Fe}] \sim+0.3$, and the few outliers with low $[\alpha / \mathrm{Fe}]$ do not constitute a trend of alpha abundance with metallicity.

We seek to better quantify these trends, accounting for the different sample sizes. We thus choose three partially overlapping metallicity bins, $-2.5<[\mathrm{Fe} / \mathrm{H}]<-1.5,-2.0<$ $[\mathrm{Fe} / \mathrm{H}]<-1.0,-1.5<[\mathrm{Fe} / \mathrm{H}]<-0.5$. We do not include higher or lower metallicities due to the lack of a correction function for $[\alpha / \mathrm{Fe}]_{\mathrm{atm}}$, and a paucity of measured abundances, respectively. For each metallicity bin, we measure the average alpha abundance ratio in each M31 dwarf galaxy, as well as the MW comparison sample, as described in $\S 4.1$ For this comparison, we also add literature abundances for the Sagittarius dSph (gray pentagons) from Carretta et al. (2010) and the Small Magellanic Cloud (gray hexagons) from Mucciarelli (2014, priv. comm.). Not all dwarf galaxies populate each bin, due to the different iron abundance ranges spanned by each system. Figure 6 plots the resulting $\langle[\alpha / \mathrm{Fe}]\rangle$ trend with luminosity for each metallicity bin, only for galaxies with three or more stars in each bin. Four out of five M 31 satellites appearing in all three bins show a gentle decrease in $\langle[\alpha / \mathrm{Fe}]\rangle$ for increasingly higher metallicity bins (And II, And III, And V, and NGC 185).

In the most metal-poor bin (left panel), $\langle[\alpha / \mathrm{Fe}]\rangle$ is indistinguishable between the four MW dwarf galaxies (Sextans, Leo II, Leo I, and Fornax) and three M31 dwarf galaxies (And V, And III, And II) that overlap in absolute magnitude. The two brighter M31 satellites in the figure, And VII and NGC 185 , are slightly more enhanced by $\sim 0.1-0.2$ dex, and so is the SMC datapoint. In the intermediate metallicity bin, the same three M31 satellites, And V, And III, and And II, have $\langle[\alpha / \mathrm{Fe}]\rangle$ consistent with that of Sextans, Leo II, Leo I, and Fornax. And VII and And I have enhanced alpha abundance ratios, similar to the Sag dSph $(\langle[\alpha / \mathrm{Fe}]\rangle \sim+0.3)$. NGC 185 and the SMC have $\langle[\alpha / \mathrm{Fe}]\rangle \sim+0.2$.

We detect a larger variation in $\langle[\alpha / \mathrm{Fe}]\rangle$ at the highest metallicity bin. In this bin, And V, And III, And II, and NGC 185 have $\langle[\alpha / \mathrm{Fe}]\rangle$ consistent with the MW satellites, However, the alpha abundances for And VII and NGC 147 (and perhaps M 32 but with large uncertainties) are enhanced relative to the MW dwarf galaxies at similar luminosity. Thus, in contrast to the MW satellite population, the M31 dwarf galaxies exhibit a larger variation in alpha enhancements, in particular for $\mathrm{M}_{V}<-12$ and $[\mathrm{Fe} / \mathrm{H}] \gtrsim-1.5$. We strongly caution that these trends should not be extrapolated to fainter magnitudes.

\subsection{Radial Abundance Gradients}

Radial abundance gradients, if present, may be indicators of internal variation in the chemical evolutionary history of dwarf galaxies. We restrict our analysis of iron and alpha gradients to the five galaxies with the largest samples (NGC 185, NGC 147, And II, And VII, and And V). We test for the presence of radial abundance gradients in two ways. First, we fit linear functions to $[\mathrm{Fe} / \mathrm{H}]$ and $[\alpha / \mathrm{Fe}]$ as a function of (projected) elliptical radius. The radii are scaled to the halflight radius of each galaxy, shown in Table 1 . To consider a 


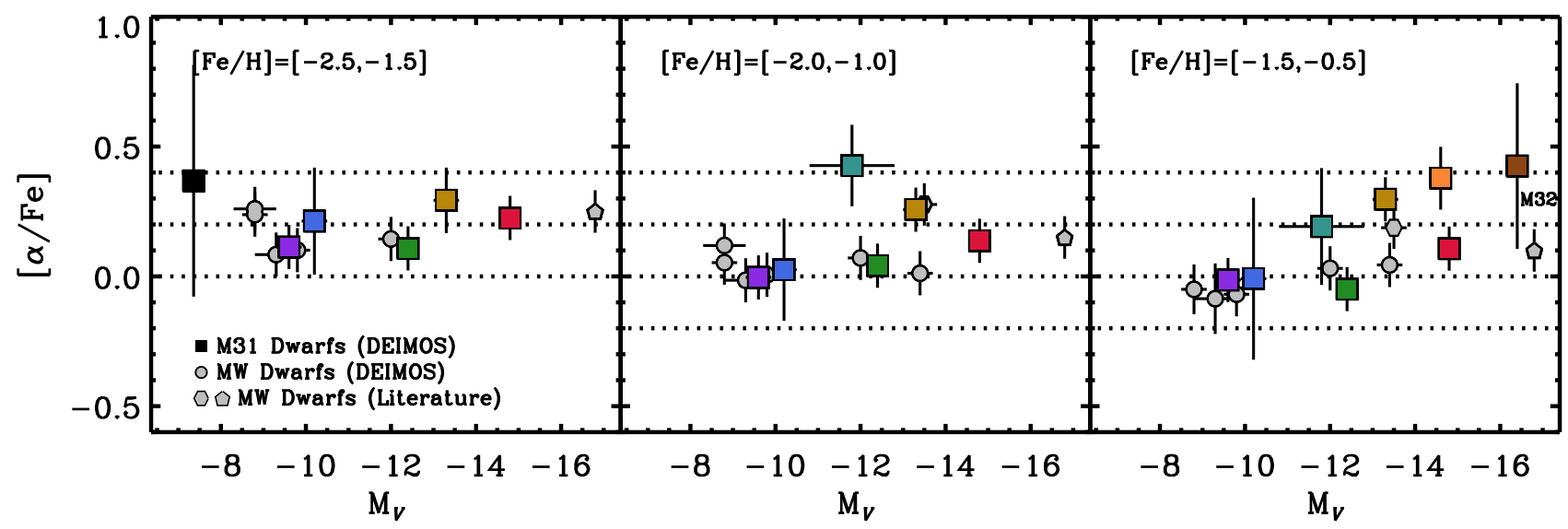

Figure 6. Variation of mean alpha abundance, $\langle[\alpha / \mathrm{Fe}]\rangle$. with luminosity as a function of $[\mathrm{Fe} / \mathrm{H}]$. We plot $\langle[\alpha / \mathrm{Fe}]\rangle$ calculated for three partially overlapping metallicity bins containing the majority of the stars in the sample. In each panel, only those dwarf galaxies with three or more $[\alpha / \mathrm{Fe}]$ measurements are shown in each panel. The color coding is the same as in Figure 5 The comparison Milky Way (MW) dwarf satellite data are shown as gray symbols. Gray circles denote MW dSphs from DEIMOS data, reanalyzed with our code. We also add literature abundances for the Sagittarius dSph (gray pentagons) from Carretta et al. (2010) and the Small Magellanic Cloud (gray hexagons) from Mucciarelli (2014). The dotted horizontal lines are added for reference in order to better distinguish changes in alpha abundance across the three metallicity bins.

Table 4

Chemical Abundance Radial Gradients

\begin{tabular}{lrrrrrr}
\hline \hline Galaxy & $\mathrm{d}[\mathrm{Fe} / \mathrm{H}] / \mathrm{d}\left(\mathrm{r} / \mathrm{r}_{1 / 2}\right)^{a}$ & $\mathrm{P}_{K S}([\mathrm{Fe} / \mathrm{H}])^{b}$ & Gradient $[\mathrm{Fe} / \mathrm{H}] ?$ & $\mathrm{~d}[\alpha / \mathrm{Fe}] / \mathrm{d}\left(\mathrm{r} / \mathrm{r}_{1 / 2}\right)^{a}$ & $\mathrm{P}_{K S}([\alpha / \mathrm{Fe}])^{b}$ & $\mathrm{Gradient}[\alpha / \mathrm{Fe}] ?$ \\
\hline NGC185 & $-0.085 \pm 0.004$ & 0.0000916 & Yes & $0.033 \pm 0.012$ & 0.0703 & Not sig. (Yes $\left.^{d}\right)$ \\
NGC147 & $0.015 \pm 0.005$ & 0.00839 & Not sig. & $0.138 \pm 0.068$ & N/A & Not sig. \\
AndVII & $-0.169 \pm 0.037$ & 0.790 & Not sig. & $0.082 \pm 0.080$ & 0.344 & Not sig. \\
AndII & $-0.448 \pm 0.023$ & 0.00000872 & Yes & $0.039 \pm 0.063$ & 0.917 & Not sig. \\
AndV & $-0.169 \pm 0.029$ & 0.479 & Not sig. & $-0.026 \pm 0.064$ & 0.993 & Not sig. \\
\hline
\end{tabular}

${ }^{a}$ Best-fit linear slope and its $1-\sigma$ uncertainty.

${ }^{\mathrm{b}} \mathrm{K}-\mathrm{S}$ probability that the inner-half and outer-half subsamples are drawn from same population.

${ }^{\mathrm{c}}$ Criteria for significance of gradient: (i) Slope has to be $3 \sigma$ away from zero, and (ii) $\mathrm{P}_{K S}<0.05$

${ }^{\mathrm{d}}$ Gradient is significant if $[\mathrm{Fe} / \mathrm{H}]>-0.5$ stars are included. This is driven by a few $[\alpha / \mathrm{Fe}]<0$ stars, see also Figure 7

gradient significant, we require that the linear slope deviate from zero by at least $3 \sigma$. We then split each galaxy's $[\mathrm{Fe} / \mathrm{H}]$ and $[\alpha / \mathrm{Fe}]$ samples in two radial bins, each subpopulation containing $50 \%$ of the stars (ordered by projected distance to the galaxy's center). Using the Kolmogorov-Smirnov (K-S) statistic, we ask what is the probability that the inner/outer $[\mathrm{Fe} / \mathrm{H}]$ and $[\alpha / \mathrm{Fe}]$ subsamples are inconsistent with being drawn from the same population with $95 \%$ confidence, i.e. $\mathrm{P}_{K S}<0.05$. We only regard as significant those gradients that pass both tests above. Given our spatial sampling, we note that the samples for And II, And V, and And VII extend roughly out to two half-light radii, while our sampling for NGC 185 and NGC 147 extends to many half-light radii.

We plot the $[\mathrm{Fe} / \mathrm{H}]$ and $[\alpha / \mathrm{Fe}]$ samples as a function of radius in Figure 7] The best-fit slopes, as well as the probability of the samples being drawn from the same population, $\mathrm{P}_{K S}$, are shown in Table 4 On the basis of the two criteria described above, we detect a significant $[\alpha / \mathrm{Fe}]$ radial gradient only in NGC 185 . One caveat is that our detection is sensitive to the inclusion of the small fraction of $[\mathrm{Fe} / \mathrm{H}]>-0.5$ stars suffering from a lack of well-tested $[\alpha / \mathrm{Fe}]-[\alpha / \mathrm{Fe}]_{\mathrm{atm}}$ calibration (see $\$ 3.2$ ); if these stars are excluded, the gradient signature is no longer significant. And VII, And V, and And II do not show any evidence for an alpha abundance gradient via either test. We do not apply the K-S test to the NGC $147[\alpha / \mathrm{Fe}]$ sample because of the limited radial coverage of the $[\alpha / \mathrm{Fe}]$ measurements. However, the slope in the outer regions is less than $3-\sigma$ away from zero. The lack of detectable $[\alpha / \mathrm{Fe}]$ radial gradients suggests that the star formation timescales were not strongly dependent on the radial distance from the center of the galaxy. In the case of And VII, high $[\alpha / \mathrm{Fe}]$ abundances dominate at all radii, suggestive of a global short star formation timescale (the few low $[\alpha / \mathrm{Fe}]$ stars are discussed in $\S 5.1$. In contrast, And V and and II have both low and high $[\alpha / \mathrm{Fe}]$ values at all radii, indicative of formation timescale long enough for a significant influence by Type Ia SNe. Out of the samples for six comparison MW dSphs, four extend beyond one half-light radius (Leo I, Leo II, Draco, and Sextans). We do not detec significant $[\alpha / \mathrm{Fe}]$ gradients in any of them.

We next consider the presence of $[\mathrm{Fe} / \mathrm{H}]$ radial gradients. We detect statistically significant radial gradients in NGC 185 and And II. Both galaxies have gradient slopes $>3 \sigma$ different from zero, and $\mathrm{P}_{K S}<<0.05$. We do not detect significant iron abundance gradients in the other three galaxies, And VII, And V and NGC 147. Our results our consistent with the findings by Ho et al. (2014) for the four galaxies in common with their sample (And V is not included). Among the four comparison MW dSphs with radial coverage larger than one halflight radius, we measure significant $[\mathrm{Fe} / \mathrm{H}]$ radial gradients in Leo I and Leo II. The presence of iron abundance gradients may be an indication of inside-out growth, radial outflows or inflows (e.g., Schroyen et al. 2013; Revaz \& Jablonka 2012).

With the possible exception of NGC 185 (see caveat above), our combined M 31 and MW sample is consistent with a lack of discernible $[\alpha / \mathrm{Fe}]$ radial gradients. In contrast, metal- 

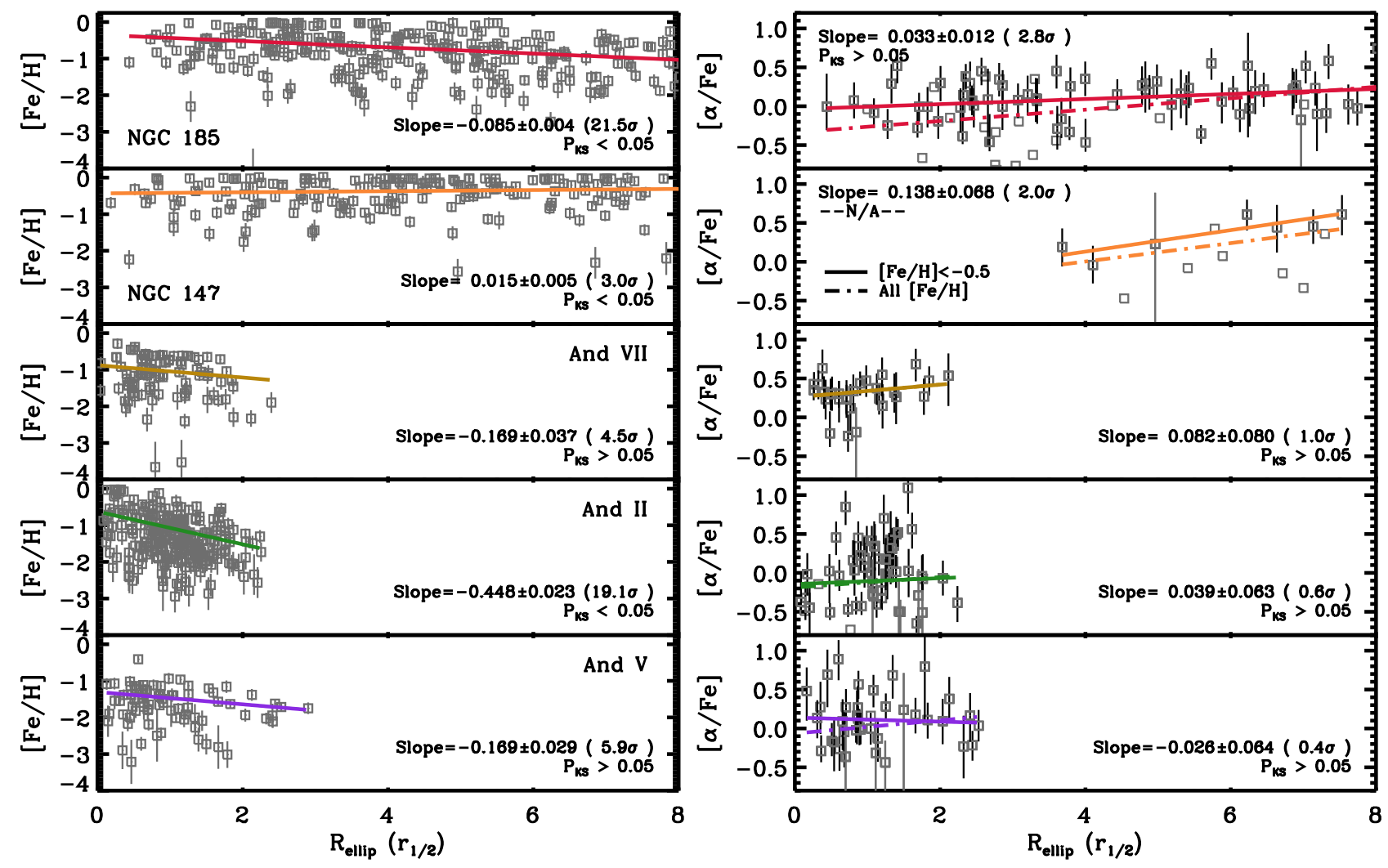

Figure 7. Radial gradients in iron and alpha abundance for the five dwarf galaxies with the largest sample sizes, shown as empty squares with $1 \sigma$ error bars. The $[\mathrm{Fe} / \mathrm{H}]$ panels show all data with $\mathrm{S} / \mathrm{N}>8 \AA^{-1}$, whereas the $[\alpha / \mathrm{Fe}]$ panels show only data with $\mathrm{S} / \mathrm{N}>15 \AA^{-1}$ (the $[\alpha / \mathrm{Fe}]$ sample). In the $[\alpha / \mathrm{Fe}]$ panels, squares with error bars indicate stars with $[\mathrm{Fe} / \mathrm{H}]<-0.5$, whereas more metal-rich objects are plotted without uncertainties. The best-fit $[\mathrm{Fe} / \mathrm{H}]$ radial gradients are plotted as solid lines, and the best-fit values are reported in each panel. The $[\alpha / \mathrm{Fe}]$ gradient fits calculated using only $[\mathrm{Fe} / \mathrm{H}]<-0.5$ data are shown as solid lines, and the slopes are also included in each panel. We also plot as dot-dashed lines the $[\alpha / \mathrm{Fe}]$ gradient fits including the $[\mathrm{Fe} / \mathrm{H}]>-0.5$ data, but caution that these $[\alpha / \mathrm{Fe}]$ abundances may not be reliable, as discussed in $\$ 3.2$ The abundances are plotted against elliptical radius. The radii have been rescaled in units of each dwarf galaxy's half-light radius, $r_{1 / 2}$, shown in Table 1 The half-light radii and gradient slopes and $1-\sigma$ uncertainties are plotted on each panel. The gradients are tabulated in Table 4

licity gradients are present in some of the dwarf galaxies of both the MW and M31. In the absence of a significant alpha abundance gradient, a metallicity gradient can still be interpreted as a difference in star formation efficiency, such that the outer, less bound regions, had greater difficulty holding on to their gas component (e.g. Lanfranchi \& Matteucci 2004), but both inner and outer regions evolved for long enough to have high and low $[\alpha / \mathrm{Fe}]$ stars. In the two M 31 satellites lacking both iron and alpha abundance gradients, the ISM may have mixed efficiently over the star formation period of the galaxy (Mac Low \& Ferrara 1999). We end with the caveat that gradients may not be visible unless data extends for many halflight radii, and our results hold for the spatial range covered by our samples. The case of Fornax in the comparison MW sample is instructive; while no gradient is detected in the central region targeted by Kirby et al. (2010), more spatially extended datasets could measure hitherto undetected abundance gradients (e.g., Leaman et al. 2013).

\section{DISCUSSION}

\subsection{Evolutionary Constraints on the M31 Dwarf Galaxies}

The combination of alpha and iron abundances serves as a star formation clock due to the different timescales between enrichment from Type II and Type Ia SNe ejecta. The M31 system contains satellite galaxies with a range of average alpha abundances from $\langle[\alpha / \mathrm{Fe}]\rangle \sim+0.0$ to $\langle[\alpha / \mathrm{Fe}]\rangle \sim$
+0.5 (see Table 3). This implies a range of star formation timescales among the M 31 dwarf galaxies. The bulk of stars in dwarf galaxies with high $[\alpha / \mathrm{Fe}]$ formed in short timescales in ISM environments rich in Type II SNe ejecta, whereas galaxies with low $[\alpha / \mathrm{Fe}]$ tended to form their stars in an ISM which was also polluted by Type Ia SNe operating on longer timescales.

We discuss the star formation timescales of M31 satellites implied from this work in combination with literature results on their stellar ages. The fainter six dwarfs galaxies were characterized as having primarily "old" stars formed $\gtrsim 10$ Gyr ago based on their horizontal branch populations (Grebel \& Guhathakurta 1999; Da Costa et al. 2002), with the possible exception of And II (Da Costa et al. 2000; McConnachie et al. 2007). Significant intermediate age populations are excluded by the absence of large numbers of asymptotic giant branch (AGB) stars (e.g., Armandroff et al. 1993; Grebel \& Guhathakurta 1999). The recent star formation histories (SFHs) by Weisz et al. (2014a) suggest that at least $50 \%$ of the stellar mass of And I, And III, and And V was in place more than $10 \mathrm{Gyr}$ ago, with the remaining stellar mass forming between $\sim 10$ and 7 Gyr ago (their Figure 7), but systematic uncertainties in the oldest age bins may lower this fraction of younger stars. In contrast, the evidence for an intermediate age stars has been confirmed by Weisz et al. (2014b), and is robust to systematic uncertainties. We discuss 
And II separately in later paragraphs due to its difference in stellar age distribution. In spite of their old(er) stellar ages, the faintest galaxies in our sample exhibit a variety of patterns in $[\alpha / \mathrm{Fe}]-[\mathrm{Fe} / \mathrm{H}]$ space. The majority have both low and high alpha abundance stars. The combination of old ages and decreasing $[\alpha / \mathrm{Fe}]$ with rising $[\mathrm{Fe} / \mathrm{H}]$ resembles the abundance patterns of some of the MW classical dSphs with old stellar populations, such as Sculptor (Tolstoy et al. 2009; Kirby et al. 2011). In the case of Sculptor, de Boer et al. (2012b) interprets this type of chemical abundance patterns as consistent with a slow burst of star formation, tapering off over a few Gyrs, and it appears that the influence of Type Ia SNe takes longer to become evident on the chemical abundances than could be naively assumed by taking the minimum age for prompt Type Ia SNe, 100 Myr (Maoz \& Mannucci 2012). Given their age and abundance similarity, the chemical evolution of the faint M31 dwarf galaxies, excluding And VII and And II, may be similar to that of the Sculptor dSph.

Among the fainter satellites, And VII has a clearly elevated characteristic alpha abundance, suggesting a short star formation timescale. The lack of a decrease in alpha abundances with rising metallicity suggests that star formation did not last long enough for Type Ia SNe to significantly enrich the ISM. Star formation may have been truncated by a close encounter with M 31 (e.g., Nichols et al. 2014) before Type Ia SNe had time to influence the alpha abundances. Given its present large separation from M31 today (> $200 \mathrm{kpc}$, McConnachie 2012), this would suggest a closer separation to M 31 in the past. Recently, Weisz et al. (2014a) found a hint of such a short SFH in their analysis of HST/WFPC2 data, but their And VII photometry was too shallow. Deeper photometry should place interesting constraints on its SFH.

In spite of And VII's overall chemical abundance trend, a few low alpha abundance outliers are present at low metallicities. The occurrence of these low $[\alpha / \mathrm{Fe}]$ stars could be the explained if And VII's abundance pattern is the sum of more than one stellar population each with different star formation characteristics. The low alpha, low $[\mathrm{Fe} / \mathrm{H}]$ stars could then be explained as coming from the secondary stellar population, e.g. from satellite-satellite accretion, or from a dissolved star cluster (e.g., Karlsson et al.2012). If so, the secondary system would have to be a smaller population in order not to dominate the overall $[\alpha / \mathrm{Fe}]-[\mathrm{Fe} / \mathrm{H}]$ abundance pattern. Example of anomalous abundance patterns are also found in other environments, such as the Milky Way halo (e.g., Ivans et al. 2003), and are interpreted similarly but in the context of halo formation. An alternate explanation is that star formation was dominated by bursts, leading to repeated enhancement in alpha from Type II SNe (Gilmore \& Wyse 1991). The presence of these low alpha stars would then be consistent with stars formed in the time between bursts, when Type Ia SNe acted to decrease $[\alpha / \mathrm{Fe}]$.

In contrast to the previously discussed satellites, And II and the brighter systems (NGC 185, NGC 147, and M 32) built their stellar populations more gradually, and reached $\sim 90 \%$ of their total mass by $\sim 5 \mathrm{Gyr}$ ago in the case of And II (Weisz et al. 2014b) and 2-4 Gyr ago for the others (Weisz et al. 2014a). Their CMD morphologies also feature red clumps, AGB populations, and in some cases carbon stars (e.g., Butler \& Martínez-Delgado 2005; Battinelli \& Demers 2004; McConnachie et al. 2007). Both And II] and NGC 185

\footnotetext{
${ }^{4}$ Recently, Amorisco et al. (2014) postulated the presence of a stream in And II confined to a region between $\mathrm{R}_{\text {And II }} \sim 0.9$ and $\sim 1.7 \mathrm{kpc}$ (projected),
}

have low average alpha abundances at a large range of metallicities. NGC 147, while having a high average alpha abundance for $[\mathrm{Fe} / \mathrm{H}]<-0.5$, may reach low alpha abundances for its highest metallicity stars. This pattern may imply that an abundance "knee" in NGC 147, indicative of the onset of Type Ia enrichment appears at a very high metallicity, suggesting a very efficient initial chemical enrichment. However, the alpha abundances for $[\mathrm{Fe} / \mathrm{H}]>-0.5$ are uncertain due to the lack of a calibrator sample for our $[\alpha / \mathrm{Fe}]$ correction term $(\$[3.2)$. The presence of many stars with low alpha abundances in And II, NGC 185, and NGC 147 is consistent with a long lasting star formation process. In addition, And II and NGC 185 show large scatter in their $[\alpha / \mathrm{Fe}]-[\mathrm{Fe} / \mathrm{H}]$ plane at fixed $[\mathrm{Fe} / \mathrm{H}]$. This may simply reflect some level of inhomogeneous chemical enrichment, such that not all ISM regions are polluted with the same amounts of Type II and Type Ia products. An alternate way to explain this scatter may be to postulate the presence of more than one track in $[\alpha / \mathrm{Fe}]-$ $[\mathrm{Fe} / \mathrm{H}]$ space, thus boosting the scatter at fixed metallicity. If so, these populations should be mixed radially, in order to be consistent with the lack of radial alpha abundance gradients discussed in $\S 4.3$. One way to probe this is by checking whether there are age differences between the high and low alpha abundance stars at a fixed metallicity.

\subsection{The Present Day M31 Dwarfs and the Build-up of M31's Halo}

In $\Lambda$ CDM, stellar halos are expected to form at least in part through accretion of smaller systems (e.g., Searle \& Zinn 1978; Bullock \& Johnston 2005; Bell et al. 2008; Cooper et al. 2010; Font et al. 2011). The chemical abundance patterns of these accreted systems thus survive as imprints in the present day stellar halo. While the metallicity of the M 31 halo has been the subject of recent work (e.g., Reitzel \& Guhathakurta 2002; Gilbert et al. 2006; Koch et al. 2008), the only stellar halo for which $[\alpha / \mathrm{Fe}]$ measurements are available is that of the MW, so we limit our comparisons to this population. The distribution of chemical abundances in MW inner halo stars disagrees with that of the present day MW dSphs (Venn et al. 2004, V13). The inner halo stars likely formed earlier than $10 \mathrm{Gyr}$ ago (e.g., Jofré \& Weiss 2011; Schuster et al. 2012; Kalirai 2012). Motivated by the differences in the M 31 dwarf satellite population relative to the MW satellites, and the fact that some of the M 31 satellites appear to be have old stellar populations systems, we now ask what type of halo would be formed out of the present day M31 dwarf galaxies, and how similar such a halo would be to what is seen in the MW.

We thus combine our dwarf galaxy samples into a single $[\alpha / \mathrm{Fe}]-[\mathrm{Fe} / \mathrm{H}]$ distribution. However, simply looking at the raw distributions of stars in our sample is incorrect. This is because the variable crowding of stars and limited size of our slitmasks prevents a uniform sampling of the M $\dot{3} 1$ satellites. Hence, we weight each star to take into account the the stellar mass of each galaxy $\left(M_{*, j}\right)$, and the luminosity function of M31 galaxies. We assign a weight to each star in our sample for galaxy $j$, using Equation 1, where the index $j$ represents the sample's galaxies ordered by luminosity.

excluding a small range of projected angles. We have compared the distribution of stellar $[\alpha / \mathrm{Fe}]$ and $[\mathrm{Fe} / \mathrm{H}]$ between their high-likelihood stream and non-stream members (priv. comm.) but do not detect a difference in the chemistries. 


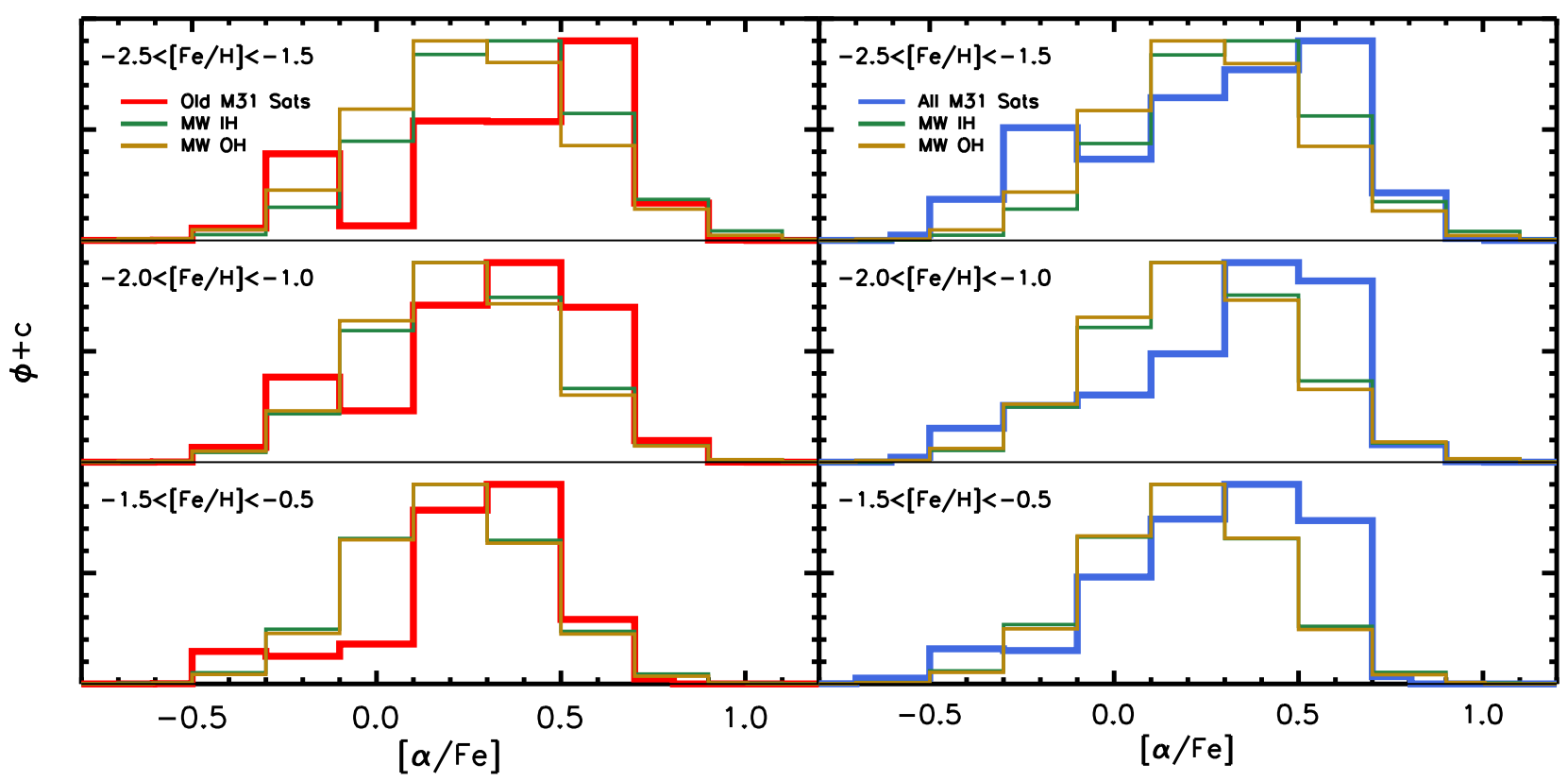

Figure 8. Comparison of the (normalized) $[\alpha / \mathrm{Fe}]$ distribution of a hypothetical stellar halo made up of M 31 satellites, as described in $\S 5.2$ against the Milky Way (MW) halo sample of Ishigaki et al. (2012). The M $31[\alpha / \mathrm{Fe}]$ distribution in the left panels (thick red histograms) includes only M 31 satellites with old stellar populations, whereas the right panels includes all satellites (thick blue histograms). The MW halo sample is split into kinematically-selected inner (thin green) and outer (thin gold) halo components. From top to bottom, we include only stars in the three metallic bins denoted in each panel. The distribution of $[\alpha / \mathrm{Fe}]$ for the M 31 satellite samples disagrees with that of the MW samples both by having a lower mean value, and a more skewed distribution, Thus, that a stellar halo made up of present day M 31 satellites could not form a MW-like halo, even if including only dwarf satellites with dominant old stellar populations.

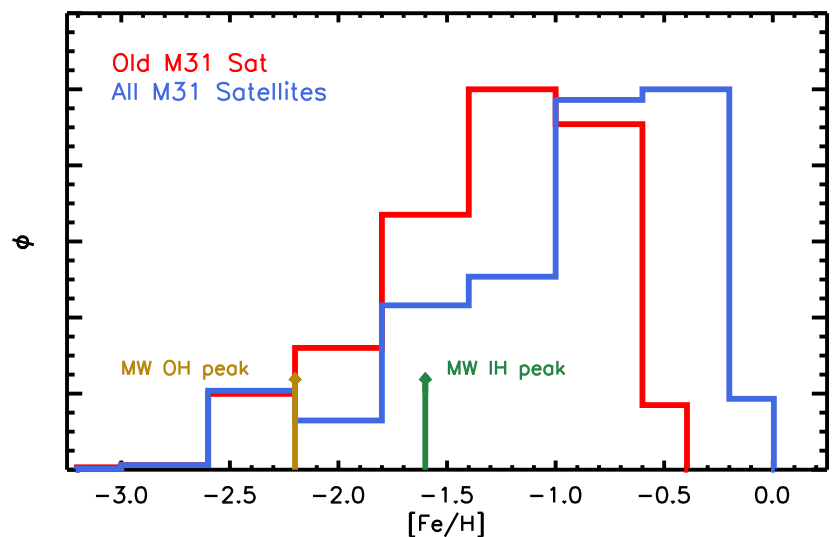

Figure 9. Normalized metallicity distributions for a stellar halo made up of destroyed M 31 satellites (same colors as in Figure 8). The arrows indicate the peaks of the metallicity distributions for the Milky Way inner and outer halos, from Carollo et al. (2010). A halo made up of present-day M 31 satellites would be too iron-rich in contrast to either MW stellar halo population, even if only considering the M 31 dwarf satellites with a majority old population.

$$
w_{i, j}=\frac{M_{*, j}\left[\Phi\left(L>0.5\left(L_{j}+L_{j-1}\right)\right)-\Phi\left(L>0.5\left(L_{j}+L_{j+1}\right)\right]\right.}{N_{j}}
$$

Here, $\Phi\left(L>L_{x}\right)$ is the cumulative luminosity function of M31 dwarf galaxies brighter than a given luminosity, and $N_{j}$ is the size of the sample for galaxy $j$. For the M 31 dwarf satellite luminosity function, we assume a simple functional form with two broken linear slopes, which roughly approximates the trend presented in the left panel of Figure 1 by Yniguez et al. (2014). We then use the weights $w$ to create $w$ samples of each star, each with the same $[\mathrm{Fe} / \mathrm{H}]$ and $[\alpha / \mathrm{Fe}]$ values as the original stars. This corrects for our non-uniform sampling of each dwarf galaxy's stars, as well as the fact that we have a larger fraction of the bright M31 satellites in our sample, but only a few of the faint ones. We have excluded M32 from all following comparisons because we lack a representative sample (only five stars), and because it is a very unusual object (Howley et al. 2013), so that its contribution to a stellar halo may not be important in most cases.

We compare the distribution of alpha abundances between the combined M 31 satellite sample and the present day MW halo as a function of metallicity. We choose the same metallicity bins as in Figure 6 For the MW halo comparison sample, we use the very high-resolution data by Ishigaki et al. (2012), who subdivide their population into inner and outer halo populations based on stellar kinematics. We only use stars in that sample for which all of $[\mathrm{Mg} / \mathrm{Fe}],[\mathrm{Si} / \mathrm{Fe}],[\mathrm{Ca} / \mathrm{Fe}]$, and $[\mathrm{Ti} \mathrm{I} / \mathrm{Fe}]$ are measured . We define $[\alpha / \mathrm{Fe}]$ for the MW sample as the unweighted mean of all four abundance ratios, consistent with the definition for our sample (see $\S 3.2$ ). The typical uncertainty in $[\alpha / \mathrm{Fe}]$ for the halo stars, $\sim 0.05 \mathrm{dex}$, is smaller than for our M31 measurements. For a more appropriate M31-MW comparison, we add Gaussian noise to each individual MW halo $[\alpha / \mathrm{Fe}]$ value, such that their typical $[\alpha / \mathrm{Fe}]$ uncertainty is then comparable to that of M 31 satellite stars. We create $\sim 1000$ realizations of each MW halo $[\alpha / \mathrm{Fe}]$ measurement using different random seeds.

Figure 8 shows histograms of the distribution in $[\alpha / \mathrm{Fe}]$ for the three metallicity bins that encompass most of our data and that of the MW stars (the same bins as in Figure 6). All histograms have been normalized to their peak value. For all three bins, both the MW inner and outer halo and M 31 satellite samples are alpha-enhanced. The peak of the M 31 samples are higher than the MW halo samples by $\sim 0.1-0.3 \mathrm{dex}$, with the difference being largest in the lowest metallicity bin. Differences in $[\alpha / \mathrm{Fe}]$ between the inner/outer MW halo have

\footnotetext{
${ }^{5}$ We use Ti I because our spectra also measure primarily neutral Ti lines.
} 
been reported by Roederer (2009) and Ishigaki et al. (2012), but as seen from the plot, are too small to be measurable when convolving the MW data with our typical data uncertainties. Nevertheless, we consider the inner and outer MW halo samples separately.

We formally compare the $[\alpha / \mathrm{Fe}]$ distributions by performing K-S tests between (a) the M31 satellite sample from old satellites only, (b) all satellites including those with intermediate age satellites), against the MW halo samples, (1) inner halo, and (2) outer halo. We perform this comparison at each metallicity bin, resulting in twelve comparison pairs. We use the associated $\mathrm{P}_{K S}$ values to assess whether the samples are inconsistent with being drawn from the same distribution. For each comparison, we recalculate $\mathrm{P}_{K S}$ by randomly sampling 400 subpopulations each with the same number of stars as the raw data samples (Table 2). This results in 400 estimates of $\mathrm{P}_{K S}$ for each comparison. In every comparison of MW halo versus all (i.e., old plus intermediate age) M 31 satellites, the $\mathrm{P}_{K S}$ values are less than $10^{-4}$. For the comparisons with oldonly M 31 satellites, the majority of realizations of $\mathrm{P}_{K S}$ peak at $<0.1$, in all but two of the comparisons. We conclude that the data is inconsistent with the MW halo and M31 dwarf satellite samples being drawn from the same parent distribution with more than $90 \%$ confidence, except for small regions in $[\alpha / \mathrm{Fe}]-[\mathrm{Fe} / \mathrm{H}]$ space. The overall difference in $[\alpha / \mathrm{Fe}]$ suggests that a halo made up of destroyed M 31 dwarf satellites (or satellites sharing their chemical evolution properties) could not have been the primary contributor to a MW-like halo.

We next compare the metallicity distribution from M31 satellites to that of the MW halo. Figure 9 plots the metallicity distributions. The red histogram plots the subsample of dwarf galaxies thought to be mainly old (And I, And III, And V, And VII). The blue histogram also incorporates And II, NGC 185 and NGC 147, which have moderate to strong evidence for significant intermediate age populations (see brief age discussion in $\S 5.1$. The figure clearly shows from the different histogram peaks that a halo made up of old M 31 satellites would be more metal-poor than one which includes the dwarf galaxies with intermediate age populations. This is a reflection of the metallicity-luminosity relation Kirby et al. 2013), since the satellites in our sample with intermediate populations also tend to be the most luminous. We compare the peaks of our histograms to the peak metallicities of the MW inner and outer halo from Carollo et al. (2010) as vertical lines at $[\mathrm{Fe} / \mathrm{H}]=-1.6$ and $[\mathrm{Fe} / \mathrm{H}]=-2.2$, respectively. The discrepancy of peak iron abundances strongly implies that a halo made up of satellites like those of M 31 would result in a halo population too metal-rich in comparison to the MW halo, regardless of the exclusion of satellites with intermediate age populations.

The comparison of alpha abundances and metallicity distributions shows that a MW halo could not be formed from a population of present-day M 31 satellites, even if we only consider those satellites with predominately old stellar populations. Thus, the old population dwarf galaxies that formed the MW halo were different from both the present-day "old" MW dwarf galaxies and "old" M 31 satellites, as has also been suggested in simulations by Robertson et al. (2005) in the context of the MW halo formation.

\section{CONCLUSIONS}

We have measured alpha abundance ratios and stellar metallicities for 226 individual RGB stars in nine dwarf galaxies of
M31. We first summarize the results internal to our sample:

1. The stellar populations of the M 31 dwarf galaxies show a variety of "internal" $[\alpha / \mathrm{Fe}]-[\mathrm{Fe} / \mathrm{H}]$ abundance patterns, including flat trends of $[\alpha / \mathrm{Fe}]$ with $[\mathrm{Fe} / \mathrm{H}]($ e.g., And VII), and decreasing $[\alpha / \mathrm{Fe}]$ with $[\mathrm{Fe} / \mathrm{H}]$ (e.g., And V).

2. The average alpha abundances ratios vary between galaxies from solar $\langle[\alpha / \mathrm{Fe}]\rangle \sim+0.0$, to alpha-enhanced $\langle[\alpha / \mathrm{Fe}]\rangle \sim+0.5$, indicating a range in star formation timescales.

3. The average alpha abundance ratios are not significantly correlated with galaxy luminosity, distance from M 31 (i.e., environment), stellar kinematics, or surface brightness.

4. The variation in alpha abundances between M 31 dwarf galaxies with $\mathrm{M}_{V}<-12$ is larger at higher metallicities, $[\mathrm{Fe} / \mathrm{H}] \gtrsim-1.5$.

5. We confirm significant radial iron abundance gradients in two galaxies out of the five with sufficient data (NGC 185 and And II). There is only tentative evidence for an alpha abundance radial gradient in NGC 185.

We have homogeneously compared our sample to a sample of Milky Way dwarf galaxy satellites, as well as to a literature sample of Milky Way inner and outer halo populations. We summarize the conclusions from these comparisons below:

6. For $\mathrm{M}_{V}<-12$, the $\mathrm{M} 31$ dwarf satellites show marginal evidence for larger variation in alpha abundance at fixed metallicity relative to the Milky Way satellites, especially at higher metallicities, $[\mathrm{Fe} / \mathrm{H}]>-1.5$.

7. In the absence of a sample of M31 halo alpha abundance, we compare the population of M31 satellites to the Milky Way stellar halo. A stellar halo made from disrupted M31 dwarf satellites would be more iron-rich and have a different alpha abundance distribution than the Milky Way halo. This holds both for old M 31 satellites and those with younger stellar populations. Therefore, the Milky Way stellar halo could not be formed from a population of dwarf galaxies with chemical properties like the present-day M 31 satellites.

The measurements of alpha abundances for M 31 satellites constitute an important contribution to our growing understanding of dwarf galaxies. The combination of accurate age distributions and chemical information (e.g., $[\alpha / \mathrm{Fe}]-[\mathrm{Fe} / \mathrm{H}]$ distributions, metallicity distribution functions, radial abundance gradients) will help in understanding the detailed evolution in these systems, thus expanding galactic archaeology beyond the Milky Way. Studies of M 31 galaxies are also complemented by those of nearby field dwarf galaxies at comparable distances.

With the study of the M31 dwarf galaxies (and systems at similar distances), we approach the limit of capabilities for medium-resolution spectroscopy of individual RGB stars with 8-10 meter class telescopes. Within the current telescope constraints, chemical abundance measurements of more distant stellar populations are still possible however by co-addition of spectra from stars with similar stellar parameters, as has been recently shown in the case of RGB 
stars by Yang et al. (2013), observations of very bright (but relatively young) blue and red supergiants (e.g., Venn et al. 2000; Davies et al. 2010), and from integrated light measurements of stellar populations such as globular clusters (e.g. Colucci et al. 2013). Thirty meter class telescopes now under development will greatly expand the spatial region accessible to studies of the chemical inventory of nearby galaxies from individual stars.

\section{ACKNOWLEDGEMENTS}

The authors wish to acknowledge extensive help from Evan Kirby on code development as well as many comments that helped improve the science discussion. We thank Raja Guhathakurta and the SPLASH team for helpful conversations and making the present work possible. We also acknowledge the anonymous referee for a useful report that contributed to improving this manuscript, as well as useful discussions with Nhung Ho and Ana Bonaca.

LCV was supported by the National Science Foundation Graduate Research Fellowship under Grant No. DGE-1122492. LCV and MG acknowledge support from NSF Grant AST-0908752. EJT was supported by NASA through Hubble Fellowship Grant No. 51316.01 awarded by the Space Telescope Science Institute, which is operated by the Association of Universities for Research in Astronomy, Inc., for NASA, under contract NAS 5-26555.

Facilities: Keck II (DEIMOS)

\section{REFERENCES}

Amorisco, N. C., Evans, N. W., \& van de Ven, G. 2014, Nature, 507, 335 Anders, E., \& Grevesse, N. 1989, Geochim. Cosmochim. Acta, 53, 197 Armandroff, T. E., Da Costa, G. S., Caldwell, N., \& Seitzer, P. 1993, AJ, 106, 986

Asplund, M., Grevesse, N., Sauval, A. J., \& Scott, P. 2009, ARA\&A, 47, 481

Battaglia, G., \& Starkenburg, E. 2012, A\&A, 539, A123

Battinelli, P., \& Demers, S. 2004, A\&A, 417, 479

Bell, E. F., Zucker, D. B., Belokurov, V., et al. 2008, ApJ, 680, 295

Brasseur, C. M., Martin, N. F., Rix, H.-W., et al. 2011, ApJ, 729, 23

Bressan, A., Marigo, P., Girardi, L., et al. 2012, MNRAS, 427, 127

Brown, W. R., Beers, T. C., Wilhelm, R., et al. 2008, AJ, 135, 564

Bullock, J. S., \& Johnston, K. V. 2005, ApJ, 635, 931

Butler, D. J., \& Martínez-Delgado, D. 2005, AJ, 129, 2217

Carigi, L., Hernandez, X., \& Gilmore, G. 2002, MNRAS, 334, 117

Carollo, D., Beers, T. C., Chiba, M., et al. 2010, ApJ, 712, 692

Carretta, E., Bragaglia, A., Gratton, R. G., et al. 2010, A\&A, 520, A95

Chieffi, A., \& Limongi, M. 2004, ApJ, 608, 405

Cohen, J. G., \& Huang, W. 2010, ApJ, 719, 931

Collins, M. L. M., Chapman, S. C., Rich, R. M., et al. 2013, ApJ, 768, 172

Colucci, J. E., Fernanda Durán, M., Bernstein, R. A., \& McWilliam, A. 2013, ApJ, 773, L36

Conn, A. R., Ibata, R. A., Lewis, G. F., et al. 2012, ApJ, 758, 11

Conroy, C., Graves, G. J., \& van Dokkum, P. G. 2014, ApJ, 780, 33

Cooper, A. P., Cole, S., Frenk, C. S., et al. 2010, MNRAS, 406, 744

Cooper, M. C., Newman, J. A., Davis, M., Finkbeiner, D. P., \& Gerke, B. F.

2012, spec2d: DEEP2 DEIMOS Spectral Pipeline, Astrophysics Source Code Library

Da Costa, G. S., Armandroff, T. E., \& Caldwell, N. 2002, AJ, 124, 332

Da Costa, G. S., Armandroff, T. E., Caldwell, N., \& Seitzer, P. 2000, AJ, 119,705

Davies, B., Kudritzki, R.-P., \& Figer, D. F. 2010, MNRAS, 407, 1203

de Boer, T. J. L., Tolstoy, E., Hill, V., et al. 2012a, A\&A, 544, A73

-. 2012b, A\&A, 539, A103

Dekel, A., \& Woo, J. 2003, MNRAS, 344, 1131

del Pino, A., Hidalgo, S. L., Aparicio, A., et al. 2013, MNRAS, 433, 1505

Dotter, A., Chaboyer, B., Jevremović, D., et al. 2008, ApJS, 178, 89

Faber, S. M., Phillips, A. C., Kibrick, R. I., et al. 2003, Proc. SPIE, 4841, 1657

Font, A. S., McCarthy, I. G., Crain, R. A., et al. 2011, MNRAS, 416, 2802

Frebel, A., Kirby, E. N., \& Simon, J. D. 2010, Nature, 464, 72
Gallart, C., Freedman, W. L., Aparicio, A., Bertelli, G., \& Chiosi, C. 1999, AJ, 118, 2245

Geha, M., Blanton, M. R., Yan, R., \& Tinker, J. L. 2012, ApJ, 757, 85

Geha, M., van der Marel, R. P., Guhathakurta, P., et al. 2010, ApJ, 711, 361

Geisler, D., Smith, V. V., Wallerstein, G., Gonzalez, G., \& Charbonnel, C. 2005, AJ, 129, 1428

Gilbert, K. M., Guhathakurta, P., Kalirai, J. S., et al. 2006, ApJ, 652, 1188

Gilbert, K. M., Guhathakurta, P., Beaton, R. L., et al. 2012, ApJ, 760, 76

Gilmore, G., \& Wyse, R. F. G. 1991, ApJ, 367, L55

Grebel, E. K., Gallagher, III, J. S., \& Harbeck, D. 2003, AJ, 125, 1926

Grebel, E. K., \& Guhathakurta, P. 1999, ApJ, 511, L101

Greene, J. E., Murphy, J. D., Graves, G. J., et al. 2013, ApJ, 776, 64

Hernandez, X., Gilmore, G., \& Valls-Gabaud, D. 2000, MNRAS, 317, 831

Ho, N., Geha, M., Munoz, R. R., et al. 2012, ApJ, 758, 124

Ho, N., Geha, M., Tollerud, E., et al. 2014, arXiv: astro-ph/1405.4424

Howley, K. M., Guhathakurta, P., van der Marel, R., et al. 2013, ApJ, 765, 65

Ivans, I. I., Sneden, C., James, C. R., et al. 2003, ApJ, 592, 906

Ishigaki, M. N., Chiba, M., \& Aoki, W. 2012, ApJ, 753, 64

Iwamoto, K., Brachwitz, F., Nomoto, K., et al. 1999, ApJS, 125, 439

Jofré, P., \& Weiss, A. 2011, A\&A, 533, A59

Kalirai, J. S. 2012, Nature, 486, 90

Kalirai, J. S., Beaton, R. L., Geha, M. C., et al. 2010, ApJ, 711, 671

Karlsson, T., Bland-Hawthorn, J., Freeman, K. C., \& Silk, J. 2012, ApJ, 759, 111

Kim, Y.-C., Demarque, P., Yi, S. K., \& Alexander, D. R. 2002, ApJS, 143, 499

Kirby, E. N. 2011, PASP, 123, 531

Kirby, E. N., Cohen, J. G., Guhathakurta, P., et al. 2013, ApJ, 779, 102

Kirby, E. N., Cohen, J. G., Smith, G. H., et al. 2011, ApJ, 727, 79

Kirby, E. N., Guhathakurta, P., Bolte, M., Sneden, C., \& Geha, M. C. 2009, ApJ, 705, 328

Kirby, E. N., Guhathakurta, P., \& Sneden, C. 2008, ApJ, 682, 1217

Kirby, E. N., Guhathakurta, P., Simon, J. D., et al. 2010, ApJS, 191, 352

Koch, A., McWilliam, A., Grebel, E. K., Zucker, D. B., \& Belokurov, V. 2008, ApJ, 688, L13

Lanfranchi, G. A., \& Matteucci, F. 2004, MNRAS, 351, 1338

-. 2007, A\&A, 468, 927

Leaman, R., Venn, K. A., Brooks, A. M., et al. 2013, ApJ, 767, 131

Letarte, B., Hill, V., Tolstoy, E., et al. 2010, A\&A, 523, A17

Mac Low, M.-M., \& Ferrara, A. 1999, ApJ, 513, 142

Maoz, D., \& Mannucci, F. 2012, PASA, 29, 447

Maoz, D., Mannucci, F., \& Brandt, T. D. 2012, MNRAS, 426, 3282

Martin, C., Carlin, J. L., Newberg, H. J., \& Grillmair, C. 2013, ApJ, 765, L39

Matteucci, F., Spitoni, E., Recchi, S., \& Valiante, R. 2009, A\&A, 501, 531

McConnachie, A. W., Irwin, M. J., Ibata, R. A., et al. 2009, Nature, 461, 66 McConnachie, A. W. 2012, AJ, 144, 4

McConnachie, A. W., Arimoto, N., \& Irwin, M. 2007, MNRAS, 379, 379

McWilliam, A., Wallerstein, G., \& Mottini, M. 2013, ApJ, 778, 149

Mucciarelli, A. 2014, Astronomische Nachrichten, 335, 79

Newman, J. A., Cooper, M. C., Davis, M., et al. 2013, ApJS, 208, 5

Nichols, M., Revaz, Y., \& Jablonka, P. 2014, A\&A, 564, A112

Nomoto, K., Tominaga, N., Umeda, H., Kobayashi, C., \& Maeda, K. 2006, Nuclear Physics A, 777, 424

Pompéia, L., Hill, V., Spite, M., et al. 2008, A\&A, 480, 379

Reitzel, D. B., \& Guhathakurta, P. 2002, AJ, 124, 234

Revaz, Y., \& Jablonka, P. 2012, A\&A, 538, A82

Robertson, B., Bullock, J. S., Font, A. S., Johnston, K. V., \& Hernquist, L. 2005, ApJ, 632, 872

Roederer, I. U. 2009, AJ, 137, 272

Schroyen, J., De Rijcke, S., Koleva, M., Cloet-Osselaer, A., \&

Vandenbroucke, B. 2013, MNRAS, 434, 888

Schuster, W. J., Moreno, E., Nissen, P. E., \& Pichardo, B. 2012, A\&A, 538, A21

Searle, L., \& Zinn, R. 1978, ApJ, 225, 357

Shetrone, M. D., Côté, P., \& Sargent, W. L. W. 2001, ApJ, 548, 592

Simon, J. D., \& Geha, M. 2007, ApJ, 670, 313

Starkenburg, E., Helmi, A., De Lucia, G., et al. 2013, MNRAS, 429, 725

Teyssier, M., Johnston, K. V., \& Kuhlen, M. 2012, MNRAS, 426, 1808

Thomas, D., Maraston, C., Bender, R., \& Mendes de Oliveira, C. 2005, ApJ, 621,673

Tinsley, B. M. 1979, ApJ, 229, 1046

Tollerud, E. J., Geha, M. C., Vargas, L. C., \& Bullock, J. S. 2013, ApJ, 768, 50

Tollerud, E. J., Beaton, R. L., Geha, M. C., et al. 2012, ApJ, 752, 45

Tolstoy, E., Hill, V., \& Tosi, M. 2009, ARA\&A, 47, 371 
Trager, S. C., Faber, S. M., Worthey, G., \& González, J. J. 2000, AJ, 120, 165

Vargas, L. C., Geha, M., Kirby, E. N., \& Simon, J. D. 2013, ApJ, 767, 134

Venn, K. A., Irwin, M., Shetrone, M. D., et al. 2004, AJ, 128, 1177

Venn, K. A., McCarthy, J. K., Lennon, D. J., et al. 2000, ApJ, 541, 610

Weisz, D. R., Dolphin, A. E., Skillman, E. D., et al. 2014a, ApJS, in press (arXiv: astro-ph/1404.7144)

Weisz, D. R., Skillman, E. D., Hidalgo, S. L., et al. 2014b, ApJ, in press (arXiv: astro-ph/1405.4870)
Woosley, S. E., \& Weaver, T. A. 1995, ApJS, 101, 181

Yang, L., Kirby, E. N., Guhathakurta, P., Peng, E. W., \& Cheng, L. 2013, ApJ, 768, 4

Yniguez, B., Garrison-Kimmel, S., Boylan-Kolchin, M., \& Bullock, J. S. 2014, MNRAS, 439, 73 